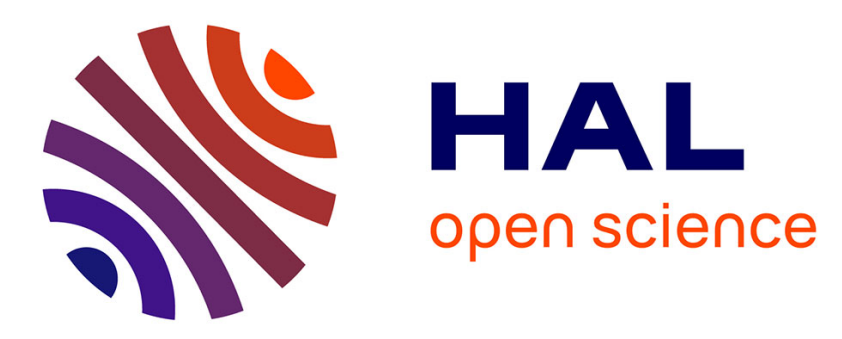

\title{
Numerical methods for an optimal multiple stopping problem
}

\author{
Imène Ben Latifa, Joseph Fréderic Bonnans, Mohamed Mnif
}

\section{To cite this version:}

Imène Ben Latifa, Joseph Fréderic Bonnans, Mohamed Mnif. Numerical methods for an optimal multiple stopping problem. Stochastics and Dynamics, 2016, 16 (4), pp.27. 10.1142/S0219493716500167 . hal-01248282

\section{HAL Id: hal-01248282 \\ https://hal.inria.fr/hal-01248282}

Submitted on 25 Dec 2015

HAL is a multi-disciplinary open access archive for the deposit and dissemination of scientific research documents, whether they are published or not. The documents may come from teaching and research institutions in France or abroad, or from public or private research centers.
L'archive ouverte pluridisciplinaire HAL, est destinée au dépôt et à la diffusion de documents scientifiques de niveau recherche, publiés ou non, émanant des établissements d'enseignement et de recherche français ou étrangers, des laboratoires publics ou privés. 


\title{
Numerical methods for an optimal multiple stopping problem
}

\author{
Imène Ben Latifa \\ ENIT-LAMSIN, University of Tunis El Manar \\ B.P. 37, 1002 Tunis-Belvédère, Tunisia. \\ benlatifa@cmap.polytechnique.fr
}

\author{
Joseph Frédéric Bonnans \\ Ecole Polytechnique-CMAP, \\ 91128 Palaiseau Cedex, France. \\ frederic.bonnans@inria.fr
}

\author{
Mohamed Mnif \\ ENIT-LAMSIN, University of Tunis El Manar \\ B.P. 37, 1002 Tunis-Belvédère, Tunisia. \\ mohamed.mnif@enit.rnu.tn
}

July 20, 2015

\begin{abstract}
This paper deals with numerical solutions to an optimal multiple stopping problem. The corresponding dynamic programming (DP) equation is a variational inequality satisfied by the value function in the viscosity sense. The convergence of the numerical scheme is shown by viscosity arguments. An optimal quantization method is used for computing the conditional expectations arising in the DP equation. Numerical results are presented for the price of swing option and the behavior of the value function.
\end{abstract}

Key words: quantization, Swing option, optimal multiple stopping time problem. 


\section{Introduction}

In their paper, Carmona and Touzi presented and implemented a numerical method to solve an optimal multiple stopping time problem by using Malliavin calculus to compute the conditional expectation arising in the DP equation and the Monte Carlo method. In this work we use the optimal quantization method and we prove the convergence of our numerical scheme to the unique viscosity solution of the associated Hamilton-JacobiBellman Variational Inequality (HJBVI in short).

Optimal quantization has been developed in the 50s in the field of Signal Processing. Its main purpose consists in approximating a continuous signal by a discrete one on an optimal way. In the 90s, its application has been extended to the field of Numerical Integration to compute some integral estimations by using finite weighted sums. And in the early 2000s, this method has been applied to the field of Numerical Probabilities and Financial Mathematics. This extension has been motivated by the necessity of designing efficient methodologies for pricing and hedging more and more sophisticated financial products. Indeed optimal quantization brought a natural answer to the conditional expectation computations appearing in these financial models (see for example Guilbaud et al. [10] for numerical methods for an optimal order execution problem).

Given a random variable $X$, a quantization algorithm should provide a finite grid $G$ and the quantized approximation of $X$ is then defined by the closest-neighbor projection of $X$ onto $G$. One of the main challenges is to compute the optimal grid ensuring that the distance between $X$ and its quantized approximation is minimal for a $L^{p}$ norm. There already exist algorithms for such purposes such as Lloyd's Method and the competitive learning vector quantization procedure $[1,9,15]$.

We fix the option maturity time $T \in(0, \infty)$, i.e. the time of expiration of our right to stop the process or exercise. Let $(\Omega, \mathcal{F}, \mathbb{P})$ be a complete probability space modeling the randomness of the market, with a filtration $\mathbb{F}=\left\{\mathcal{F}_{t}\right\}_{0 \leq t \leq T}$ satisfying the usual conditions, i.e. an increasing right continuous family of sub- $\sigma$-algebras of $\mathbb{F}$ such that $\mathcal{F}_{0}$ contains all the $\mathbb{P}$-null sets. We consider that the state process $\left(X_{t}\right)_{t \in[0, T]}$ follows the SDE:

$$
d X_{t}=b\left(X_{t}\right) d t+c\left(X_{t}\right) d W_{t}, X_{0}=x \in \mathbb{R}^{d}
$$

where $W$ denotes a d-dimensional Brownian motion defined on the probability space $(\Omega, \mathcal{F}, \mathbb{P}), b: \mathbb{R}^{d} \rightarrow \mathbb{R}^{d}$ and $c: \mathbb{R}^{d} \rightarrow \mathcal{M}(d, q)$ are Lipschitz continuous, where $\mathcal{M}(d, q)$ denotes the set of matrix with $d$ rows and $q$ columns. The value function of the swing option problem with $\ell$ exercise rights and refraction time $\delta>0$ is given by:

$$
v^{(\ell)}(x)=\sup _{\left(\tau_{1}, \ldots, \tau_{\ell}\right) \in \mathcal{S}_{0}^{(\ell)}} E^{Q}\left[\sum_{i=1}^{\ell} e^{-r \tau_{i}} \phi\left(X_{\tau_{i}}^{0, x}\right)\right],
$$


where $\phi: \mathbb{R}^{d} \longrightarrow \mathbb{R}^{+}$is a Lipschitz payoff function with linear growth, and

$$
\mathcal{S}_{0}^{(\ell)}:=\left\{\begin{array}{l}
\left(\tau_{1}, \ldots, \tau_{\ell}\right) \in \mathcal{S}_{0}^{\ell}, \tau_{1} \in \mathcal{S}_{0}, \tau_{i}-\tau_{i-1} \geq \delta \text { on }\left\{\tau_{i-1}+\delta \leq T\right\} \text { a.s, } \\
\tau_{i}=\left(T_{+}\right) \text {on }\left\{\tau_{i-1}+\delta>T\right\} \text { a.s, } \forall i=2, \ldots, \ell .
\end{array}\right\},
$$

where $\mathcal{S}_{0}$ is the set of stopping times taking values in $[0, T]$.

The optimal multiple stopping time problem (1.2) can be seen as sequential ordinary optimal stopping problems. It is known that the solution of each ordinary problem is the unique viscosity solution of the HJBVI

$$
\begin{aligned}
& \min \left\{r v^{(k)}(t, x)-\frac{\partial v^{(k)}}{\partial t}(t, x)-L v^{(k)}(t, x)\right. \\
& \left.v^{(k)}(t, x)-\phi_{v^{(k-1)}}(t, x)\right\}=0, \quad \forall(t, x) \in[0, T) \times \mathbb{R}^{d} \\
& v^{(k)}(T, x)=\phi(x) \quad \forall x \in \mathbb{R}^{d},
\end{aligned}
$$

where $\phi: \mathbb{R}^{d} \longrightarrow \mathbb{R}^{+}$is a Lipschitz payoff function, $k=1, \ldots, \ell$,

$$
\begin{gathered}
L v^{(k)}(t, x):=\frac{1}{2} \sum_{i=1}^{d} \sum_{j=1}^{d}\left(c(t, x) c^{T}(t, x)\right)_{i, j} \frac{\partial^{2} v^{(k)}}{\partial x_{i} \partial x_{j}}(t, x)+\sum_{i=1}^{d} b_{i}(t, x) \frac{\partial v^{(k)}}{\partial x_{i}}(t, x) \text { and } \\
\phi_{v^{(k-1)}}(t, x)=\phi(x)+e^{-r \delta} E\left[v^{(k-1)}\left(t+\delta, X_{t+\delta}^{t, x}\right)\right], \quad \forall 0 \leq t \leq T-\delta \\
\phi_{v^{(k-1)}}(t, x)=\phi(x), \quad \forall T-\delta<t \leq T .
\end{gathered}
$$

We refer to [4] in the jump diffusion case.

We recall the definition of the viscosity solution.

Definition 1.1 Let $k=1, \ldots, \ell$, and $u^{(k)}$ be a continuous function.

(i) We say that $u^{(k)}$ is a viscosity super-solution (sub-solution) of (1.4)-(1.5) if

$$
\min \left\{r \psi\left(t_{0}, x_{0}\right)-\frac{\partial \psi}{\partial t}\left(t_{0}, x_{0}\right)-L \psi\left(t_{0}, x_{0}\right) ; \psi\left(t_{0}, x_{0}\right)-\phi^{(k)}\left(t_{0}, x_{0}\right)\right\} \geq 0
$$

$(\leq 0)$ whenever $\psi \in \mathcal{C}^{1,2}([0, T) \times \mathbb{R}) \cap \mathcal{C}_{2}([0, T] \times \mathbb{R})$ and $u^{(k)}-\psi$ has a strict global minimum (maximum) at $\left(t_{0}, x_{0}\right) \in[0, T) \times \mathbb{R}$.

(ii) We say that $u^{(k)}$ is a viscosity solution of (1.4)-(1.5) if it is both super and sub-solution of (1.4)-(1.5).

Where

$$
\mathcal{C}_{2}([0, T] \times \mathbb{R}):=\left\{\psi \in \mathcal{C}^{0}([0, T] \times \mathbb{R}) / \sup _{[0, T] \times \mathbb{R}} \frac{|\psi(t, x)|}{1+|x|^{2}}<+\infty\right\}
$$

The optimal quantization method is used for pricing an American option in a continuoustime Markov process, which consists in solving a standard optimal stopping problem (see Bally et al. [2]). In this paper we extend their result for a multiple stopping time problem. We estimate the price of a swing option expiring at time $T$, with $\ell$ rights of exercise when 
the price process is solution of (1.1). The exact simulation of the diffusion at time $t$ is not possible. We use the Markovian discretization scheme, e.g. the Euler scheme:

$$
X_{t_{n+1}}=X_{t_{n}}+b\left(X_{t_{n}}\right) \frac{T}{M}+c\left(X_{t_{n}}\right)\left(W_{t_{n+1}}-W_{t_{n}}\right), \quad 0 \leq n \leq M-1,
$$

where $M$ is a positive integer and $t_{n}:=n T / M$.

We approximate the Snell envelope by quantization of the Euler scheme (1.8). Using Zador's Theorem we prove the convergence of our algorithm.

In one dimensional case, we consider that the price process $X=\left(X_{t}\right)_{0 \leq t \leq T}$ satisfies the following stochastic differential equation (SDE)

$$
d X_{t}=r X_{t} d t+\sigma X_{t} d W_{t}, \quad X_{0}=x>0
$$

where $W$ denotes a one-dimensional Brownian motion defined on the probability space $(\Omega, \mathcal{F}, \mathbb{P}), r>0$ and $\sigma \in \mathbb{R}$. The filtration considered here is the natural filtration of the Brownian motion completed by the $\mathbb{P}$-null sets. It is known that there exists a unique strong solution for the SDE, $X_{t}=x e^{\left(r-\frac{\sigma^{2}}{2}\right) t+\sigma W_{t}}$. So it suffices to consider a quantization of the Brownian motion itself.

The paper is organized as follows. In section 2, we recall some basic facts about optimal quantization. In section 3 , we present our numerical scheme on general multidimensional diffusion process and we prove, for some assumptions on $b$ and $c$, the convergence of our algorithm with rate $O\left(\frac{M^{1+\frac{1}{d}}}{N^{\frac{1}{d}}}\right)$, where $N:=N_{0}+\ldots+N_{M}\left(N_{n}\right.$ is the size of $\hat{X}_{n}$, the optimal discretization of $X_{n}$ ). In section 4 , we present our numerical scheme on the specific one-dimensional linear setting and we prove that our scheme is monotonous, stable and consistent and consequently, it converges to the solution of our optimal multiple stopping time problem. In section 5, we present some numerical results on one-dimensional linear setting which are similar to those of Carmona and Touzi [6], we discuss the complexity of the algorithm and we end by a comparison of our method with the finite difference one.

\section{Background on optimal quantization}

We begin with a brief introduction to optimal quantization of random variable [2] (see Graf and Luschgy [8] for an overview). Let $(\Omega, \mathcal{F}, \mathbb{P})$ be a probability space and let $X$ be a random variable with distribution $\mathbb{P}_{X}$. Assume that $X \in L^{p}$, optimal $L^{p}$ mean quantization $(p>1)$ with level $N$ consists in studying the best $L^{p}$ approximation of $X$ by some random variable $\hat{X}=q(X)$, where $q: \mathbb{R}^{d} \rightarrow \mathbb{R}^{d}$ is a Borel function taking at most $\mathrm{N}$ values. We associate to every Borel function $q(X)$ taking at most $N$ values, the $L^{p}$-mean quantization error $\|X-q(X)\|_{p}$ measuring the distance between the two random vectors $X$ and $q(X)$ w.r.t. the mean $L^{p}$-norm, where $\|X\|_{p}:=\left(E|X|^{p}\right)^{1 / p}$ and $|$.$| denotes$ 
an arbitrary norm on $R^{d}$. Minimizing the $L^{p}$ mean quantization error, $\|X-q(X)\|_{p}$, can be decomposed into two successive phases:

1. Let $\Gamma=\left(x_{1}, \ldots, x_{N}\right) \in\left(\mathbb{R}^{d}\right)^{N}$, find a quantizer $q_{\Gamma}: \mathbb{R}^{d} \rightarrow x_{1}, \ldots, x_{N}$ such that

$$
\left\|X-q_{\Gamma}(X)\right\|_{p}=\inf \left\{\|X-q(X)\|_{p}, q: \mathbb{R}^{d} \rightarrow x_{1}, \ldots, x_{N}, \text { Borel function }\right\} .
$$

2. Find an $N$-tuple $\Gamma^{*} \in\left(\mathbb{R}^{d}\right)^{N}$ that achieves the infimum of $\left\|X-\hat{X}^{\Gamma}\right\|_{p}$ over $\left(\mathbb{R}^{d}\right)^{N}$, that is

$$
\left\|X-\hat{X}^{\Gamma^{*}}\right\|_{p}=\inf \left\{\left\|X-\hat{X}^{\Gamma}\right\|_{p}, \Gamma \in\left(\mathbb{R}^{d}\right)^{N}\right\},
$$

where $\hat{X}^{\Gamma}=q_{\Gamma}(X)$ and

$$
\begin{aligned}
\left\|X-\hat{X}^{\Gamma}\right\|_{p}^{p} & =\sum_{i=1}^{N} E\left[\mathbf{1}_{C_{i}(\Gamma)}\left|X-x_{i}\right|^{p}\right]=E\left[\min _{1 \leq i \leq N}\left|X-x_{i}\right|^{p}\right] \\
& =\int_{\mathbb{R}^{d}} \min _{1 \leq i \leq N}\left|x_{i}-y\right|^{p} \mathbb{P}_{X}(d y) .
\end{aligned}
$$

The solution of the first optimization problem, denoted $q_{\Gamma}$, is the closest neighbor projections induced by the Voronoi tessellations of $u$ as defined below.

Definition 2.1 (i) Let $\Gamma=\left(x_{1}, \ldots, x_{N}\right) \in\left(\mathbb{R}^{d}\right)^{N}$. A Borel partition $C_{i}(\Gamma), i=1, \ldots, N$, of $\mathbb{R}^{d}$ is a Voronoi tessellation of $u$ if for every $i \in 1, \ldots, N, C_{i}(\Gamma)$ satisfies

$$
C_{i}(\Gamma) \subset\left\{y \in \mathbb{R}^{d},\left|x_{i}-y\right|=\min _{1 \leq j \leq N}\left|x_{j}-y\right|\right\} .
$$

(ii) The closest neighbor projection or Voronoi quantizer (function) $q_{\Gamma}$ induced by the Voronoi tessellation $\left(C_{i}(\Gamma)\right)_{1 \leq i \leq N}$ is defined, for every $y \in \mathbb{R}^{d}$, by $q_{\Gamma}(y)=\sum_{1 \leq i \leq N} x_{i} \mathbf{1}_{C_{i}(\Gamma)}(y)$.

(iii) The random variable

$$
\hat{X}^{\Gamma}=q_{\Gamma}(X)=\sum_{1 \leq i \leq N} x_{i} \mathbf{1}_{C_{i}(\Gamma)}(X)
$$

is called a Voronoi quantization of $X$. The $N$-tuple $\Gamma$ is often called an $N$-quantizer.

One shows (see [2]) that the solution of the second problem (2.1) is given by

$$
\left\|X-\hat{X}^{\Gamma^{*}}\right\|_{p}=\min \left\{\|X-Z\|_{p}, Z: \Omega \rightarrow \mathbb{R}^{d}, \text { random variable },|Z(\Omega)| \leq N\right\},
$$

and $\hat{X}^{\Gamma^{*}}$ is called an optimal quantizer of $X$. We can see that when the grids are optimal (in the quadratic quantization sense), the spatial order of convergence is better then that obtained with usual grid methods. Zador's Theorem (see [7], [5] and [8]) says that

$$
\left\|X-\hat{X}^{x^{*}}\right\|_{p}=C_{X, p} N^{-1 / d}+o\left(N^{-1 / d}\right) .
$$




\section{Multi-dimentional diffusion process}

First, let us give some notations that will be used throughout this paper.

\section{Notations:}

- |.| will denote the canonical Euclidean norm on $\mathbb{R}^{d}$.

- $\mathcal{M}(d, q)$ will denote the set of matrix with $d$ rows and $q$ columns.

- For every matrix $A \in \mathcal{M}(d, q)$, set $\|A\|^{2}:=\operatorname{Tr}\left(A A^{*}\right.$ ) (which corresponds to its Euclidean norm as an element of $\left.\mathbb{R}^{d \times q}\right)$.

- For a Lipschitz continuous function $f,[f]_{\text {Lip }}$ denotes the Lipschitz constant of $\phi: \mathbb{R}^{d} \rightarrow \mathbb{R}$.

The process $X$ is the diffusion process solution of the stochastic differential equation

$$
X_{t}=x+\int_{0}^{t} b\left(X_{s}\right) d s+\int_{0}^{t} c\left(X_{s}\right) d W_{s}, x \in \mathbb{R}^{d}
$$

where $b: \mathbb{R}^{d} \rightarrow \mathbb{R}^{d}$ and $c: \mathbb{R}^{d} \rightarrow \mathcal{M}(d, q)$ are Lipschitz continuous, there exists a positive constant $C^{\text {Lip }}$ such that

$$
\left\|c(x)-c\left(x^{\prime}\right)\right\|+\left|b(x)-b\left(x^{\prime}\right)\right| \leq C^{L i p}\left|x-x^{\prime}\right|, \quad \forall\left(x, x^{\prime}\right) \in \mathbb{R}^{d} \times \mathbb{R}^{d} .
$$

Note that $b$ and $c$ have at most linear growth. We will assume that this real constant $C^{\text {Lip }}$ also satisfies

$$
\|c(x)\|+|b(x)| \leq C^{L i p}(1+|x|), \quad \forall x \in \mathbb{R}^{d} .
$$

It is known that for all $p \geq 1$, there exists a positive constant $C_{p, T}$ such that

$$
E\left(\sup _{t \in[0, T]}\left|X_{t}\right|^{p}\right) \leq C_{p, T}\left(1+|x|^{p}\right)
$$

Let us define the following HJBVI

$$
\begin{aligned}
& \min \left\{r v^{(k)}(t, x)-\frac{\partial v^{(k)}}{\partial t}(t, x)-L v^{(k)}(t, x) ;\right. \\
& \left.v^{(k)}(t, x)-\phi_{v^{(k-1)}}(t, x)\right\}=0, \quad \forall(t, x) \in[0, T) \times \mathbb{R}^{d} \\
& v^{(k)}(T, x)=\phi(x) \quad \forall x \in \mathbb{R}^{d},
\end{aligned}
$$

where $\phi: \mathbb{R}^{d} \longrightarrow \mathbb{R}^{+}$is a Lipschitz payoff function, $k=1, \ldots, \ell$,

$$
\begin{gathered}
L v^{(k)}(t, x):=\frac{1}{2} \sum_{i=1}^{d} \sum_{j=1}^{d}\left(c(t, x) c^{T}(t, x)\right)_{i, j} \frac{\partial^{2} v^{(k)}}{\partial x_{i} \partial x_{j}}(t, x)+\sum_{i=1}^{d} b_{i}(t, x) \frac{\partial v^{(k)}}{\partial x_{i}}(t, x) \text { and } \\
\phi_{v^{(k-1)}}(t, x)=\phi(x)+e^{-r \delta} E\left[v^{(k-1)}\left(t+\delta, X_{t+\delta}^{t, x}\right)\right], \quad \forall 0 \leq t \leq T-\delta \\
\phi_{v^{(k-1)}}(t, x)=\phi(x), \quad \forall T-\delta<t \leq T .
\end{gathered}
$$


Assume that $v^{(0)}=0$ and for all $t>T, v^{(k)}\left(t, X_{t}\right)=0$.

For $k=1, \ldots, \ell$, we have that the solution of the following stopping time problem

$$
v^{(k)}(t, x)=\operatorname{ess} \sup _{\tau \in \mathcal{S}_{t}} E\left[e^{-r(\tau-t)} \phi_{v^{(k-1)}}\left(\tau, X_{\tau}\right) \mid X_{t}=x\right],
$$

is the viscosity solution of the HJBVI (3.4)-(3.5), where $\mathcal{S}_{t}:=\left\{\tau \in \mathcal{S}_{0} ; t \leq \tau \leq T\right\}$ for every $t \in \mathcal{S}_{0}$.

\subsection{Numerical scheme}

Time discretization consists in approximating the continuous time diffusion $\left(X_{t}\right)_{t \in[0, T]}$ by the following Euler scheme. Set $t_{n}:=n T / M$, where $M \geq 1$ is an integer, the Euler scheme is defined by

$$
\bar{X}_{t_{n+1}}=\bar{X}_{t_{n}}+b\left(\bar{X}_{t_{n}}\right) \frac{T}{M}+c\left(\bar{X}_{t_{n}}\right)\left(W_{t_{n+1}}-W_{t_{n}}\right), \quad \bar{X}_{0}=x .
$$

We denote by $P^{n, i}$ the transition from $\bar{X}_{n}$ to $\bar{X}_{n+i}, P^{n, i}(v)(x):=E\left[v\left(\bar{X}_{n+i}\right) \mid \bar{X}_{n}=x\right]$, where $v$ is a function from $\mathbb{R}^{d}$ to $\mathbb{R}$. We have that the transition $P^{n, i}$ is Lipschitz in the following sense: for every Lipschitz continuous function $f: \mathbb{R}^{d} \rightarrow \mathbb{R}$

$$
\left[P^{n, i} f\right]_{L i p}\left(1+C_{b, c, T} T / M\right)[f]_{L i p},
$$

where $C_{b, c, T}$ is a positive constant depending on $b, c$ and $T$, (see, e.g., [1] for a proof).

For $k=1, \ldots, \ell, n=0, \ldots, M$, let $V_{n}^{(k)}$ the solution of

$$
V_{n}^{(k)}=\operatorname{ess} \sup _{\tau \in \Theta_{n}} E\left[e^{-r\left(\tau-t_{n}\right)} \phi_{V^{(k-1)}}\left(\tau, \bar{X}_{\tau}\right) \mid \mathcal{F}_{t_{n}}\right],
$$

where $\Theta_{n}$ denotes the set of $\left\{t_{n}, \ldots, t_{M}\right\}$-valued $\left(\mathcal{F}_{t_{u}}\right)_{u \in\{n, \ldots, M\}}$ stopping times. It satisfies the so-called backward programming formula (see [13]):

$$
\left\{\begin{aligned}
V_{M}^{(k)} & :=\phi\left(\bar{X}_{M}\right), \\
V_{n}^{(k)} & :=\max \left\{e^{-r h} E\left[V_{n+1}^{(k)} \mid \mathcal{F}_{t_{n}}\right], \phi_{V_{n}^{(k-1)}}\left(\bar{X}_{n}\right)\right\}, \quad 0 \leq n \leq M-1,
\end{aligned}\right.
$$

where

$$
\begin{aligned}
& \phi_{V_{n}^{(k-1)}}\left(\bar{X}_{n}\right)=\phi\left(\bar{X}_{n}\right)+e^{-r \delta} E\left[V_{n+i_{0}}^{(k-1)} \mid \mathcal{F}_{t_{n}}\right], \quad 0 \leq n \leq M-i_{0}, \\
& \phi_{V_{n}^{(k-1)}}\left(\bar{X}_{n}\right)=\phi\left(\bar{X}_{n}\right), \quad M-i_{0}<n \leq M-1 .
\end{aligned}
$$

$i_{0}$ is an integer such that $\delta=i_{0} T / M$.

Using the Markov property, we derive a dynamic programming formula in distribution: for $k=1, \ldots, \ell, V_{n}^{(k)}=v_{n}^{(k)}\left(\bar{X}_{n}\right), n \in\{0, \ldots, M\}$, where the function $v_{n}^{(k)}$ is solution of

$$
\left\{\begin{aligned}
v_{M}^{(k)} & :=\phi, \\
v_{n}^{(k)} & :=\max \left\{e^{-r h} P^{n, 1}\left(v_{n+1}^{(k)}\right), \phi_{v_{n}^{(k-1)}}\right\}, \quad 0 \leq n \leq M-1,
\end{aligned}\right.
$$


where for $x \in \mathbb{R}^{d}$,

$$
\begin{aligned}
& \phi_{v_{n}^{(k-1)}}(x)=\phi(x)+e^{-r \delta} E\left[v_{n+i_{0}}^{(k-1)} \mid X_{n}=x\right], \quad 0 \leq n \leq M-i_{0}, \\
& \phi_{v_{n}^{(k-1)}}(x)=\phi(x), \quad M-i_{0}<n \leq M .
\end{aligned}
$$

We assume that $v_{n}^{(k)} \equiv 0$ if $n>M$ and $v_{n}^{(0)} \equiv 0$, for $n=0, \ldots, M$.

Let us prove the Lipschitz property of the function $v_{n}^{(k)}$ for $k=1, \ldots, \ell$ and $n=0, \ldots, M$.

Proposition 3.1 For $k=1, \ldots, \ell, n=0, \ldots, M, v_{n}^{(k)}$ is Lipschitz continuous function and there exists a positive constant $C$ such that

$$
\left[v_{n}^{(k)}\right]_{\text {Lip }} \leq C[\phi]_{\text {Lip }}
$$

Proof. We will prove this proposition by induction in $k$.

For $k=1$, we have that $v_{M}^{(1)}=\phi$, then $v_{M}^{(1)}$ is Lipschitz continuous function and $\left[v_{M}^{(1)}\right]_{\text {Lip }}=$ $[\phi]_{\text {Lip. }}$. Using the inequality

$$
|\max (a, b)-\max (c, d)| \leq \max (|a-c|,|b-d|)
$$

one concludes by induction in $n$ that, for $n=0, \ldots, M, v_{n}^{(1)}$ is Lipschitz continuous function and

$\left[v_{n}^{(1)}\right]_{\text {Lip }} \leq C[\phi]_{\text {Lip }}$.

Let $1 \leq k \leq \ell-1$, assume that for $n=0, \ldots, M, v_{n}^{(k)}$ is Lipschitz continuous function with $\left[v_{n}^{(k)}\right]_{\text {Lip }} \leq C[\phi]_{\text {Lip }}$ and let us prove that it is also for $v_{n}^{(k+1)}$.

For $n=M$, we have that $v_{M}^{(k+1)}=\phi$, then $v_{M}^{(k+1)}$ is Lipschitz continuous function and $\left[v_{M}^{(k+1)}\right]_{\text {Lip }}=[\phi]_{\text {Lip }}$.

Let $M-i_{0}+1 \leq n \leq M$, assume that $v_{n+1}^{(k+1)}$ is Lipschitz continuous function with $\left[v_{n+1}^{(k+1)}\right]_{\text {Lip }} \leq C[\phi]_{\text {Lip }}$ and let us prove that it is also for $v_{n}^{(k+1)}$. Using the Lipschitz property of $P^{n, 1}$, we have that for $x, y \in \mathbb{R}^{d}$

$$
\begin{aligned}
\left|v_{n}^{(k+1)}(x)-v_{n}^{(k+1)}(y)\right| & \leq \max \left\{[\phi]_{\text {Lip }}, C\left[v_{n+1}^{(k+1)}\right]_{\text {Lip }}\right\}|x-y| \\
& \leq C[\phi]_{\text {Lip }}|x-y| .
\end{aligned}
$$

Then, for $M-i_{0}+1 \leq n \leq M, v_{n}^{(k+1)}$ is Lipschitz continuous function with $\left[v_{n}^{(k+1)}\right]_{\text {Lip }} \leq$ $C[\phi]_{\text {Lip }}$.

Now, let $0 \leq n \leq M-i_{0}$, assume that $v_{n+1}^{(k+1)}$ is Lipschitz continuous function with $\left[v_{n+1}^{(k+1)}\right]_{\text {Lip }} \leq C[\phi]_{\text {Lip }}$ and let us prove that it is also for $v_{n}^{(k+1)}$. Using the Lipschitz property of $P^{n, 1}$ and $P^{n, i_{0}}$, we have that for $x, y \in \mathbb{R}^{d}$

$$
\begin{aligned}
\left|v_{n}^{(k+1)}(x)-v_{n}^{(k+1)}(y)\right| & \leq \max \left\{\left([\phi]_{\text {Lip }}+C\left[v_{n+i_{0}}^{(k)}\right]_{\text {Lip }}\right), C\left[v_{n+1}^{(k+1)}\right]_{\text {Lip }}\right\}|x-y| \\
& \leq C[\phi]_{\text {Lip }}|x-y| .
\end{aligned}
$$


We conclude that for $k=1, \ldots, \ell$ and $n=M, \ldots, 0, v_{n}^{(k)}$ is Lipschitz continuous function with $\left[v_{n}^{(k)}\right]_{\text {Lip }} \leq C[\phi]_{\text {Lip }}$.

Now we discretize the random variable $\bar{X}_{t_{n}}$ by some $\sigma\left(\bar{X}_{t_{n}}\right)$-random variable $\hat{X}_{n}$ taking finitely many values in $\mathbb{R}^{d}$. $\hat{X}_{n}$ is the optimal quantization of $\bar{X}_{t_{n}}$. Let $N:=N_{0}+\ldots+N_{M}$ denote the total number of elementary quantizers used to quantize the whole Markov chain $\left(\bar{X}_{t_{n}}\right)_{0 \leq n \leq M}$. We denote by $\left\{x_{1}^{n}, \ldots, x_{N_{n}}^{n}\right\}=q_{n}\left(\mathbb{R}^{d}\right)$ the grid of $N_{n}$ points used to quantize $\bar{X}_{t_{n}}$, and by $x^{n}:=\left(x_{1}^{n}, \ldots, x_{N_{n}}^{n}\right)$ the induced $N_{n}$-tuple. Note that $\bar{X}_{0}=x$, so that $\hat{X}_{0}=x$ is the best possible $L^{p}$-mean quantization of $\bar{X}_{0}$ and $N_{0}=1$.

We replace $\bar{X}_{n}$ in (3.11) by its quantized random vector $\hat{X}_{n}$ and we obtain the quantized dynamic programming formula

$$
\left\{\begin{array}{l}
\hat{V}_{M}^{(k)}=\phi\left(\hat{X}_{M}\right), \\
\hat{V}_{n}^{(k)}=\max \left\{e^{-r h} E\left[\hat{V}_{n+1}^{(k)} \mid \hat{X}_{n}\right], \hat{\phi}_{\hat{V}_{n}^{(k-1)}}\left(\hat{X}_{n}\right)\right\}, \quad 0 \leq n \leq M-1,
\end{array}\right.
$$

where

$$
\begin{aligned}
& \hat{\phi}_{\hat{V}_{n}^{(k-1)}}\left(\hat{X}_{n}\right)=\phi\left(\hat{X}_{n}\right)+e^{-r \delta} E\left[\hat{V}_{n+i_{0}}^{(k-1)} \mid \hat{X}_{n}\right], \quad 0 \leq n \leq M-i_{0}, \\
& \hat{\phi}_{\hat{V}_{n}^{(k-1)}}\left(\hat{X}_{n}\right)=\phi\left(\hat{X}_{n}\right), \quad M-i_{0}<n \leq M .
\end{aligned}
$$

For simplicity of notation we denote by $\hat{E}_{n}[]:.=E\left[. \mid \hat{X}_{n}\right]$ and $E_{n}[]:.=E\left[. \mid X_{n}\right]$, and we assume that $V_{n}^{(k)} \equiv \hat{V}_{n}^{(k)} \equiv 0$ for $n>M$.

We define the function $\hat{v}_{n}^{(k)}$ by

$$
\left\{\begin{array}{l}
\hat{v}_{M}^{(k)}\left(x_{i}^{M}\right)=\phi\left(x_{i}^{M}\right), \forall i \in\left\{1, \ldots, N_{M}\right\} \\
\hat{v}_{n}^{(k)}\left(x_{i}^{n}\right)=\max \left\{e^{-r h} \sum_{j=1}^{N_{n+1}} \pi_{i j}^{n, 1} \hat{v}_{n+1}^{(k)}\left(x_{j}^{n+1}\right), \phi_{\hat{v}^{(k-1)}}^{N}\left(t_{n}, x_{i}^{n}\right)\right\}, 1 \leq i \leq N_{n}, 0 \leq n \leq M-1,
\end{array}\right.
$$

where

$$
\begin{aligned}
& \phi_{\hat{v}^{(k-1)}}^{N}\left(t_{n}, x_{i}^{n}\right)=\phi\left(x_{i}^{n}\right)+e^{-r \delta} \sum_{j=1}^{N_{n+i_{0}}} \pi_{i j}^{n, i_{0}} \hat{v}_{n+i_{0}}^{(k-1)}\left(x_{j}^{n+i_{0}}\right), \quad 1 \leq i \leq N_{n}, 0 \leq n \leq M-i_{0}, \\
& \phi_{\hat{v}^{(k-1)}}^{N}\left(t_{n}, x_{i}^{n}\right)=\phi\left(x_{i}^{n}\right), \quad 1 \leq i \leq N_{n}, M-i_{0}<n \leq M,
\end{aligned}
$$

and for $m \in\left\{1, i_{0}\right\}, i=1, \ldots, N_{n}, j=1, \ldots, N_{n+1}, n=0, \ldots, M-m$

$$
\begin{aligned}
\pi_{i j}^{n, m} & :=\mathbb{P}\left(\hat{X}_{n+m}=x_{j}^{n+m} \mid \hat{X}_{n}=x_{i}^{n}\right)=\mathbb{P}\left(\bar{X}_{n+m} \in C_{j}\left(x^{n+m}\right) \mid \bar{X}_{n} \in C_{i}\left(x^{n}\right)\right) \\
& =\frac{p_{i j}^{n, m}}{p_{i}^{m}} \text { with } p_{i j}^{n, m}:=\mathbb{P}\left(\bar{X}_{n+m} \in C_{j}\left(x^{n+m}\right), \bar{X}_{n} \in C_{i}\left(x^{n}\right)\right) \text { and } p_{i}^{m}:=\mathbb{P}\left(\bar{X}_{n} \in C_{i}\left(x^{n}\right)\right) .
\end{aligned}
$$

Assume that $\hat{v}^{(0)} \equiv 0$. We can see that $\hat{V}_{n}^{(k)}=\hat{v}_{n}^{(k)}\left(\hat{X}_{n}\right)$.

Let us now move to the convergence of our method. 


\subsection{Convergence of the quantization method}

In this paragraph we show some a priori $L^{p}$-error bounds for $\left\|V_{n}^{(k)}-\hat{V}_{n}^{(k)}\right\|_{p}, k=1, \ldots, \ell$ and $n=0, \ldots, M$, based on the $L^{p}$-mean quantization errors $\left\|\bar{X}_{n}-\hat{X}_{n}\right\|_{p}, n=0, \ldots, M$.

Theorem 3.1 Let $\left(\hat{V}_{n}^{(k)}\right)_{0 \leq n \leq M}$ and $\left(V_{n}^{(k)}\right)_{0 \leq n \leq M}, k=1, \ldots, \ell$, be like in (3.11) and (3.13) respectively. For every $n=0, \ldots, M$, let $\hat{X}_{n}$ denote the quantization of $\bar{X}_{n}$. Then, for every $p \geq 1$ there exists a real positive constant $C$ such that

$$
\left\|V_{n}^{(k)}-\hat{V}_{n}^{(k)}\right\|_{p} \leq C \sum_{i=n}^{M}\left\|\bar{X}_{i}-\hat{X}_{i}\right\|_{p} .
$$

Proof. For $k=1, \ldots, \ell, n=0, \ldots, M$ and $j$ a strictly positive integer, set $\psi_{n, j}^{(k)}:=$ $P^{n, j}\left(v_{n+j}^{(k)}\right)$. This function satisfies

$$
E\left[v_{n+j}^{(k)}\left(\bar{X}_{n+j}\right) \mid \mathcal{F}_{t_{n}}\right]=E_{n}\left[v_{n+j}^{(k)}\left(\bar{X}_{n+j}\right)\right]=\psi_{n, j}^{(k)}\left(\bar{X}_{n}\right)
$$

It is known from inequality $(3.9)$ that $\psi_{n, j}^{(k)}$ is Lipschitz continuous function and $\left[\psi_{n, j}^{(k)}\right]_{\text {Lip }} \leq$ $C\left[v_{n+j}^{(k)}\right]_{\text {Lip }}$.

We similarly define $\hat{\psi}_{n, j}^{(k)}$ by the equality $\hat{\psi}_{n, j}^{(k)}\left(\hat{X}_{n}\right):=\hat{E}_{n}\left[\hat{v}_{n+j}^{(k)}\left(\hat{X}_{n+j}\right)\right]$.

First step: For $k=1$.

For $n=M$ we have that $V_{M}^{(1)}=\phi$, then $V_{M}^{(1)}$ is Lipschitz continuous function and $\left[V_{M}^{(1)}\right]_{\text {Lip }}=[\phi]_{\text {Lip }}$.

For $n=0, \ldots, M$,

$$
\begin{aligned}
\left|V_{n}^{(1)}-\hat{V}_{n}^{(1)}\right| & \leq\left|\phi\left(\bar{X}_{n}\right)-\phi\left(\hat{X}_{n}\right)\right|+\left|\psi_{n, 1}^{(1)}\left(\bar{X}_{n}\right)-\hat{\psi}_{n, 1}^{(1)}\left(\hat{X}_{n}\right)\right| \\
& \leq[\phi]_{L i p}\left|\bar{X}_{n}-\hat{X}_{n}\right|+\left|\psi_{n, 1}^{(1)}\left(\bar{X}_{n}\right)-\hat{E}_{n}\left[\psi_{n, 1}^{(1)}\left(\bar{X}_{n}\right)\right]\right|+\left|\hat{E}_{n}\left[\psi_{n, 1}^{(1)}\left(\bar{X}_{n}\right)\right]-\hat{\psi}_{n, 1}^{(1)}\left(\hat{X}_{n}\right)\right|
\end{aligned}
$$

On the other hand, we have

$$
\begin{gathered}
\left|\psi_{n, 1}^{(1)}\left(\bar{X}_{n}\right)-\hat{E}_{n}\left[\psi_{n, 1}^{(1)}\left(\bar{X}_{n}\right)\right]\right| \leq\left|\psi_{n, 1}^{(1)}\left(\bar{X}_{n}\right)-\psi_{n, 1}^{(1)}\left(\hat{X}_{n}\right)\right|+\hat{E}_{n}\left|\psi_{n, 1}^{(1)}\left(\hat{X}_{n}\right)-\psi_{n, 1}^{(1)}\left(\bar{X}_{n}\right)\right| \\
\leq C\left[v_{n+1}^{(1)}\right]_{L i p}\left(\left|\bar{X}_{n}-\hat{X}_{n}\right|+\hat{E}_{n}\left|\bar{X}_{n}-\hat{X}_{n}\right|\right)
\end{gathered}
$$

then

$$
\left\|\psi_{n, 1}^{(1)}\left(\bar{X}_{n}\right)-\hat{E}_{n}\left[\psi_{n, 1}^{(1)}\left(\bar{X}_{n}\right)\right]\right\|_{p} \leq C\left[v_{n+1}^{(1)}\right]_{L i p}\left\|\bar{X}_{n}-\hat{X}_{n}\right\|_{p}
$$

By using the tower property of the conditional expectation and since $\hat{X}_{n}$ is $\sigma\left(\bar{X}_{n}\right)$ measurable, we have

$$
\begin{aligned}
\hat{E}_{n}\left[\psi_{n, 1}^{(1)}\left(\bar{X}_{n}\right)\right]-\hat{\psi}_{n, 1}^{(1)}\left(\hat{X}_{n}\right) & =\hat{E}_{n}\left[E_{n}\left[v_{n+1}^{(1)}\left(\bar{X}_{n+1}\right)\right]\right]-\hat{E}_{n}\left[\hat{v}_{n+1}^{(1)}\left(\hat{X}_{n+1}\right)\right] \\
& =\hat{E}_{n}\left[v_{n+1}^{(1)}\left(\bar{X}_{n+1}\right)-\hat{v}_{n+1}^{(1)}\left(\hat{X}_{n+1}\right)\right] .
\end{aligned}
$$


Then,

$$
\left\|\hat{E}_{n}\left[\psi_{n, 1}^{(1)}\left(\bar{X}_{n}\right)\right]-\hat{\psi}_{n, 1}^{(1)}\left(\hat{X}_{n}\right)\right\|_{p} \leq\left\|V_{n+1}^{(1)}-\hat{V}_{n+1}^{(1)}\right\|_{p}
$$

Finally, it follows from the above inequalities and (3.16) that for $n \in\{0, \ldots, M\}$

$$
\begin{aligned}
\left\|V_{n}^{(1)}-\hat{V}_{n}^{(1)}\right\|_{p} & \leq\left([\phi]_{L i p}+C\left[v_{n+1}^{(1)}\right]_{L i p}\right)\left\|\bar{X}_{n}-\hat{X}_{n}\right\|_{p}+\left\|V_{n+1}^{(1)}-\hat{V}_{n+1}^{(1)}\right\|_{p} \\
& \leq C[\phi]_{L i p}\left\|\bar{X}_{n}-\hat{X}_{n}\right\|_{p}+\left\|V_{n+1}^{(1)}-\hat{V}_{n+1}^{(1)}\right\|_{p}
\end{aligned}
$$

The last inequality is deduced from $v_{n+1}^{(1)}$.

On the other hand, $\left\|V_{M}^{(1)}-\hat{V}_{M}^{(1)}\right\|_{p} \leq[\phi]_{L i p}\left\|\bar{X}_{n}-\hat{X}_{n}\right\|_{p}$, so that

$$
\left\|V_{n}^{(1)}-\hat{V}_{n}^{(1)}\right\|_{p} \leq C[\phi]_{\text {Lip }} \sum_{i=n}^{M}\left\|\bar{X}_{i}-\hat{X}_{i}\right\|_{p}
$$

Second step: Let $1 \leq k \leq \ell-1$, assume that $\left\|V_{n}^{(k)}-\hat{V}_{n}^{(k)}\right\|_{p} \leq C[\phi]_{\text {Lip }} \sum_{i=n}^{M}\left\|\bar{X}_{i}-\hat{X}_{i}\right\|_{p}$, and let us prove that it is true for $k+1$.

We have that $V_{M}^{(k+1)}=\phi$, then $V_{M}^{(k+1)}$ is Lipschitz continuous function and $\left[V_{M}^{(k+1)}\right]_{L i p}=$ $[\phi]_{\text {Lip }}$.

For $n=M, \ldots, 0$, we have

$$
\left|V_{n}^{(k+1)}-\hat{V}_{n}^{(k+1)}\right| \leq\left|\psi_{n, 1}^{(k+1)}\left(\bar{X}_{n}\right)-\hat{\psi}_{n, 1}^{(k+1)}\left(\hat{X}_{n}\right)\right|+\left|\phi_{V_{n}^{(k)}}\left(\bar{X}_{n}\right)-\hat{\phi}_{\hat{V}_{n}^{(k)}}\left(\hat{X}_{n}\right)\right| .
$$

As it is done in the first step, we obtain that

$$
\begin{aligned}
\left\|\psi_{n, 1}^{(k+1)}\left(\bar{X}_{n}\right)-\hat{\psi}_{n, 1}^{(k+1)}\left(\hat{X}_{n}\right)\right\|_{p} & \leq C\left[\psi_{n, 1}^{(k+1)}\right]_{L i p}\left\|\bar{X}_{n}-\hat{X}_{n}\right\|_{p}+\left\|V_{n+1}^{(k+1)}-\hat{V}_{n+1}^{(k+1)}\right\|_{p} \\
& \leq C[\phi]_{L i p}\left\|\bar{X}_{n}-\hat{X}_{n}\right\|_{p}+\left\|V_{n+1}^{(k+1)}-\hat{V}_{n+1}^{(k+1)}\right\|_{p} .
\end{aligned}
$$

According to the definitions of $\phi_{V_{n}^{(k)}}$ and $\hat{\phi}_{\hat{V}_{n}^{(k)}}$, we have that

$$
\begin{gathered}
\left|\phi_{V_{n}^{(k)}}\left(\bar{X}_{n}\right)-\hat{\phi}_{\hat{V}_{n}^{(k)}}\left(\hat{X}_{n}\right)\right| \leq[\phi]_{L i p}\left|\bar{X}_{n}-\hat{X}_{n}\right|+\left|\psi_{n, i_{0}}^{(k)}\left(\bar{X}_{n}\right)-\hat{E}_{n}\left[\psi_{n, i_{0}}^{(k)}\left(\bar{X}_{n}\right)\right]\right| \\
+\left|\hat{E}_{n}\left[\psi_{n, i_{0}}^{(k)}\left(\bar{X}_{n}\right)\right]-\hat{\psi}_{n, i_{0}}^{(k)}\left(\hat{X}_{n}\right)\right| .
\end{gathered}
$$

On the other hand, we have

$$
\begin{aligned}
\left|\psi_{n, i_{0}}^{(k)}\left(\bar{X}_{n}\right)-\hat{E}_{n}\left[\psi_{n, i_{0}}^{(k)}\left(\bar{X}_{n}\right)\right]\right| & \leq\left|\psi_{n, i_{0}}^{(k)}\left(\bar{X}_{n}\right)-\psi_{n, i_{0}}^{(k)}\left(\hat{X}_{n}\right)\right|+\left|\psi_{n, i_{0}}^{(k)}\left(\hat{X}_{n}\right)-\hat{E}_{n}\left[\psi_{n, i_{0}}^{(k)}\left(\bar{X}_{n}\right)\right]\right| \\
& \leq\left[\psi_{n, i_{0}}^{(k)}\right]_{L i p}\left(\left|\bar{X}_{n}-\hat{X}_{n}\right|+\hat{E}_{n}\left|\bar{X}_{n}-\hat{X}_{n}\right|\right)
\end{aligned}
$$

then

$$
\begin{aligned}
\left\|\psi_{n, i_{0}}^{(k)}\left(\bar{X}_{n}\right)-\hat{E}_{n}\left[\psi_{n, i_{0}}^{(k)}\left(\bar{X}_{n}\right)\right]\right\|_{p} & \leq C\left[v_{n+i_{0}}^{(k)}\right]_{L i p}\left\|\bar{X}_{n}-\hat{X}_{n}\right\|_{p} \\
& \leq C[\phi]_{L i p}\left\|\bar{X}_{n}-\hat{X}_{n}\right\|_{p}
\end{aligned}
$$


By using the tower property of the conditional expectation and since $\hat{X}_{n}$ is $\sigma\left(X_{n}\right)$ measurable, we have

$$
\begin{aligned}
\hat{E}_{n}\left[\psi_{n, i_{0}}^{(k)}\left(\bar{X}_{n}\right)\right]-\hat{\psi}_{n, i_{0}}^{(k)}\left(\hat{X}_{n}\right) & =\hat{E}_{n}\left[E_{n}\left[v_{n+i_{0}}^{(k)}\left(X_{n+i_{0}}\right)\right]\right]-\hat{E}_{n}\left[\hat{v}_{n+i_{0}}^{(k)}\left(\hat{X}_{n+i_{0}}\right)\right] \\
& =\hat{E}_{n}\left[v_{n+i_{0}}^{(k)}\left(X_{n+i_{0}}\right)-\hat{v}_{n+i_{0}}^{(k)}\left(\hat{X}_{n+i_{0}}\right)\right] .
\end{aligned}
$$

Then

$$
\left\|\hat{E}_{n}\left[\psi_{n, i_{0}}^{(k)}\left(\bar{X}_{n}\right)\right]-\hat{\psi}_{n, i_{0}}^{(k)}\left(\hat{X}_{n}\right)\right\|_{p} \leq\left\|V_{n+i_{0}}^{(k)}-\hat{V}_{n+i_{0}}^{(k)}\right\|_{p}
$$

From inequalities (3.19), (3.20), (3.21), (3.22) and (3.23), we obtain that

$$
\left\|V_{n}^{(k+1)}-\hat{V}_{n}^{(k+1)}\right\|_{p} \leq C[\phi]_{L i p}\left\|\bar{X}_{n}-\hat{X}_{n}\right\|_{p}+\left\|V_{n+1}^{(k+1)}-\hat{V}_{n+1}^{(k+1)}\right\|_{p}+\left\|V_{n+i_{0}}^{(k)}-\hat{V}_{n+i_{0}}^{(k)}\right\|_{p}
$$

From the induction hypothesis we obtain that

$$
\begin{aligned}
\left\|V_{n}^{(k+1)}-\hat{V}_{n}^{(k+1)}\right\|_{p} & \leq C[\phi]_{\text {Lip }}\left\|\bar{X}_{n}-\hat{X}_{n}\right\|_{p}+\left\|V_{n+1}^{(k+1)}-\hat{V}_{n+1}^{(k+1)}\right\|_{p}+C[\phi]_{\text {Lip }} \sum_{i=n}^{M}\left\|\bar{X}_{i}-\hat{X}_{i}\right\|_{p} \\
& \leq C[\phi]_{\text {Lip }} \sum_{i=n}^{M}\left\|\bar{X}_{i}-\hat{X}_{i}\right\|_{p}+\left\|V_{n+1}^{(k+1)}-\hat{V}_{n+1}^{(k+1)}\right\|_{p} .
\end{aligned}
$$

From the definition of $V_{M}^{(k+1)}$ and $\hat{V}_{M}^{(k+1)}$, we have that $\left\|V_{M}^{(k+1)}-\hat{V}_{M}^{(k+1)}\right\|_{p} \leq C[\phi]_{L i p}\left\|\bar{X}_{M}-\hat{X}_{M}\right\|_{p}$ and the result follows, i.e. $\left\|V_{n}^{(k+1)}-\hat{V}_{n}^{(k+1)}\right\|_{p} \leq C[\phi]_{\text {Lip }} \sum_{i=n}^{M}\left\|\bar{X}_{i}-\hat{X}_{i}\right\|_{p}$.

Remark 3.1 Bally and Pagès introduced the following assumption. they assumed that the $L^{p}$-mean quantization errors of the $\bar{X}_{n}$ are $\varphi$-dominated, i.e. there exists a random vector $R \in L^{p+\eta}(\mathbb{P})(\eta>0)$ and a sequence $\left(\varphi_{m, n}\right)_{0 \leq m \leq n<\infty}$ such that, for every $n \geq 1$, every $m \in\{0, \ldots, n\}$ and every $N \geq 1$,

$$
\left\|\bar{X}_{m}-\hat{X}_{m}\right\|_{p} \leq \varphi_{m, n}\|R-\hat{R}\|_{p}
$$

It is shown in [2, Theorem 4] that: We assume that $\left(\bar{X}_{n}\right)_{0 \leq n \leq M}$ is $\varphi$-dominated in the sense of (3.24) by $\varphi_{n, M}:=c \sqrt{n / M}$. Let $N \geq M+1$, assume that for every $n \in\{1, \ldots, M\}$,

$$
N_{n}=\left|\hat{X}_{k}(\Omega)\right|:=\left\lceil\frac{t_{n}^{\frac{d}{2(d+1)}}(N-1)}{t_{1}^{\frac{d}{2(d+1)}}+\ldots+t_{n}^{\frac{d}{2(d+1)}}+\ldots+t_{M}^{\frac{d}{2(d+1)}}}\right\rceil,
$$

where $\lceil x\rceil:=\min \{k \in \mathbb{N} \mid k \geq x\}$. Then, there exists a positive constant $C_{p}$ such that

$$
\max _{0 \leq n \leq M}\left\|V_{n}^{(k)}-\hat{V}_{n}^{(k)}\right\|_{p} \leq C_{p} \frac{M^{1+\frac{1}{d}}}{N^{\frac{1}{d}}} .
$$




\section{Numerical scheme for one-dimensional case}

From this section we will study the one-dimensional case. We define the process $X=\left(X_{t}\right)_{0 \leq t \leq T}$ which evolves according to the following stochastic differential equation:

$$
d X_{t}=r X_{t} d t+\sigma X_{t} d W_{t}, \quad X_{0}=x>0,
$$

where $W$ is a standard Brownian motion, $r>0$ and $\sigma \in \mathbb{R}$. We shall also use the notation $X_{s}^{t, x}$ for $X_{s}$ whenever we need to emphasize the dependence of the process $X$ on its initial condition. Let us define the following HJBVI

$$
\begin{aligned}
& \min \left\{r v^{(k)}(t, x)-\frac{\partial v^{(k)}}{\partial t}(t, x)-\frac{1}{2} \sigma^{2} x^{2} \frac{\partial^{2} v^{(k)}}{\partial x^{2}}(t, x)-r x \frac{\partial v^{(k)}}{\partial x}(t, x) ;\right. \\
& \left.v^{(k)}(t, x)-\phi_{v^{(k-1)}}(t, x)\right\}=0, \quad \forall(t, x) \in[0, T) \times(0, \infty) \\
& v^{(k)}(T, x)=\phi(x) \quad \forall x \in(0, \infty),
\end{aligned}
$$

where $\phi: \mathbb{R} \longrightarrow \mathbb{R}^{+}$is a Lipschitz payoff function (in later use we define $\phi(x)=(K-x)_{+}$), $k=1, \ldots, \ell$ and

$$
\begin{aligned}
& \phi_{v^{(k-1)}}(t, x)=\phi(x)+e^{-r \delta} E\left[v^{(k-1)}\left(t+\delta, X_{t+\delta}^{t, x}\right)\right], \quad \forall 0 \leq t \leq T-\delta \\
& \phi_{v^{(k-1)}}(t, x)=\phi(x), \quad \forall T-\delta<t \leq T .
\end{aligned}
$$

Assume that $v^{(0)}=0$ and for all $t>T, v^{(k)}\left(t, X_{t}\right)=0$.

For $k=1, \ldots, \ell$, we have that the solution of the following stopping time problem

$$
v^{(k)}(t, x)=\operatorname{ess} \sup _{\tau \in \mathcal{S}_{t}} E\left[e^{-r(\tau-t)} \phi_{v^{(k-1)}}\left(\tau, X_{\tau}\right) \mid X_{t}=x\right],
$$

is the viscosity solution of the HJBVI (4.2)-(4.3), where $\mathcal{S}_{t}:=\left\{\tau \in \mathcal{S}_{0} ; t \leq \tau \leq T\right\}$ for every $t \in \mathcal{S}_{0}$. We can see that the diffusion process $\left(X_{s}\right)$, solution of (4.1), is a function of the Brownian motion, defined by

$$
X_{s}=x e^{\left(r-\frac{\sigma^{2}}{2}\right)(s-t)+\sigma W_{s-t}}, \text { for } s>t, \text { where } X_{t}=x .
$$

Then, it suffices to consider a quantizer of the Brownian motion. Let $U$ be a standard normal random variable, then we can write

$$
X_{s} \sim x e^{\left(r-\frac{\sigma^{2}}{2}\right)(s-t)+\sigma \sqrt{s-t} U}, \text { for } s>t, \text { where } X_{t}=x .
$$

Note that the optimal quantization has no closed formula for the solutions. Quadratic optimal N-quantization of the $\mathcal{N}\left(0, I_{d}\right)$ distributions has been carried out systematically for various sizes $N \in\{1, \ldots, 400\}$ and dimensions $d \in\{1, \ldots, 10\}$. Some files of these optimal grids (including their weights) can be downloaded from the website [17].

Let $\left(u_{1}, \ldots, u_{N}\right) N$ real variables, $\hat{U}$ the optimal quantizer of $U$ with values in $\left(u_{1}, \ldots, u_{N}\right)$ and

$$
\hat{X}_{s}^{t, x}=x e^{\left(r-\frac{\sigma^{2}}{2}\right)(s-t)+\sigma \sqrt{s-t} \hat{U}}, \text { for } s>t \text {, where } \hat{X}_{t}^{t, x}=x \text {. }
$$


We define

$$
\begin{aligned}
& P_{R}: \mathbb{R} \longrightarrow[0, R] \\
& \quad x \longrightarrow x \mathbf{1}_{[0, R]}+R \mathbf{1}_{(R, \infty)}, \\
& \phi_{\hat{v}_{h}^{N-R-1)}}^{N, R}(t, x)=\phi(x)+e^{-r \delta} E\left[\hat{v}_{h}^{(k-1)}\left(t+\delta, P_{R}\left(\hat{X}_{t+\delta}^{t, x}\right)\right)\right] \quad \forall t \in[0, T-\delta], \\
& \phi_{\hat{v}_{h}^{N, R-1)}}^{N, R}(t, x)=\phi(x) \quad \forall t \in(T-\delta, T] .
\end{aligned}
$$

We denote by $\hat{v}_{h}^{(k)}$, the solution of

$$
\left\{\begin{array}{l}
\hat{v}_{h}^{(k)}(t, x)=\phi(x) \quad \forall t \in[T-h, T] \\
\hat{v}_{h}^{(k)}(t, x)=\max \left\{e^{-r h} E\left[\hat{v}_{h}^{(k)}\left(t+h, P_{R}\left(\hat{X}_{t+h}^{t, x}\right)\right)\right], \phi_{\hat{v}_{h}^{(k-1)}}^{N, R}(t, x)\right\} \quad \forall t \in[0, T-h) .
\end{array}\right.
$$

Assume that $\hat{v}_{h}^{(0)} \equiv 0$ and $\hat{v}_{h}^{(k)}(t, x)=0$ for all $t>T$. We denote the numerical scheme of the HJBVI (4.2)-(4.3) by

$$
\begin{aligned}
& S^{h, N, R, k}\left(t, x, \hat{v}_{h}^{(k)}(t, x), \hat{v}_{h}^{(k)}, \hat{v}_{h}^{(k-1)}\right)=0 \quad \forall(t, x) \in[0, T-h) \times(0, \infty) \\
& \hat{v}_{h}^{(k)}(t, x)=\phi(x) \quad \forall(t, x) \in[T-h, T] \times(0, \infty),
\end{aligned}
$$

where

$$
S^{h, N, R, k}\left(t, x, p_{k}, a_{k}, a_{k-1}\right):=\min \left\{\frac{p_{k}-e^{-r h} E\left[a_{k}\left(t+h, P_{R}\left(\hat{X}_{t+h}^{t, x}\right)\right)\right]}{h} ; p_{k}-\phi_{a_{k-1}}^{N, R}(t, x)\right\} .
$$

The following proposition shows the Lipschitz property of $\hat{v}_{h}^{(k)}, k=1, \ldots, \ell$ and an upper bound of the corresponding Lipschitz constant.

Proposition 4.1 For $k=1, \ldots, \ell$, the solution $\hat{v}_{h}^{(k)}$ of (4.7)-(4.8) is Lipschitz with respect to the state variable and

$$
\left[\hat{v}_{h}^{(k)}(t, .)\right]_{\text {Lip }} \leq\left(\sum_{j=0}^{k-1} e^{j(T+\delta) \frac{\sigma^{2}}{2}}\right) e^{\frac{\sigma^{2}}{2} T}[\phi]_{\text {Lip }}, \quad \text { where } \quad[\phi]_{\text {Lip }}:=\sup _{x \neq y} \frac{|\phi(x)-\phi(y)|}{x-y}
$$

Proof. For $k=1$, by the Lipschitz assumption of $\phi$ we have that for $t \in[T-h, T]$, $\hat{v}_{h}^{(1)}(t,$.$) is Lipschitz and \left[\hat{v}_{h}^{(k)}(t, .)\right]_{\text {Lip }}=[\phi]_{\text {Lip }}$. For $t \in[T-2 h, T-h)$ we have

$$
\begin{aligned}
& \left|\hat{v}_{h}^{(1)}(t, x)-\hat{v}_{h}^{(1)}(t, y)\right| \\
& \leq \max \left(e^{-r h} E\left[\left|\hat{v}_{h}^{(1)}\left(t+h, P_{R}\left(\hat{X}_{t+h}^{t, x}\right)\right)-\hat{v}_{h}^{(1)}\left(t+h, P_{R}\left(\hat{X}_{t+h}^{t, y}\right)\right)\right|\right] ;|\phi(x)-\phi(y)|\right) \\
& \leq[\phi]_{\text {Lip }} \max \left(e^{-r h} E\left[\left|P_{R}\left(\hat{X}_{t+h}^{t, x}\right)-P_{R}\left(\hat{X}_{t+h}^{t, y}\right)\right|\right] ;|x-y|\right) \\
& \leq[\phi]_{\text {Lip }} \max \left(e^{-r h} E\left[\left|\hat{X}_{t+h}^{t, x}-\hat{X}_{t+h}^{t, y}\right|\right] ;|x-y|\right) \\
& \leq[\phi]_{\text {Lip }} \max \left(e^{-r h}|x-y| E\left[e^{\left(r-\frac{\sigma^{2}}{2}\right) h+\sigma \sqrt{h} \hat{U}}\right] ;|x-y|\right) \\
& \leq[\phi]_{\text {Lip }} e^{\frac{\sigma^{2}}{2} h}|x-y|,
\end{aligned}
$$


where the last inequality is deduced from the fact that $\hat{U}$ is the optimal quantizer of $U$, then $E\left[e^{\sigma \sqrt{h} \hat{U}}\right]=E\left[e^{\sigma \sqrt{h} E[U \mid \hat{U}]}\right]$, and using Jensen's inequality we obtain

$$
\begin{gathered}
E\left[e^{\sigma \sqrt{h} E[U \mid \hat{U}]}\right] \leq E\left[E\left[e^{\sigma \sqrt{h} U} \mid \hat{U}\right]\right]=E\left[e^{\sigma \sqrt{h} U}\right]=e^{\sigma^{2} h}, \text { so } \\
E\left[e^{\sigma \sqrt{h} \hat{U}}\right] \leq e^{\sigma^{2} h}
\end{gathered}
$$

By repeating the same reasoning for $t$ on each interval of the form $[T-(i+1) h, T-i h)$, for $i=2, \ldots, M-1$, we obtain that $\hat{v}_{h}^{(1)}(t,$.$) is also Lipschitz and \left[\hat{v}_{h}^{(1)}(t, .)\right]_{\text {Lip }} \leq e^{\frac{\sigma^{2}}{2} T}[\phi]_{\text {Lip }}$. Let $1 \leq k \leq \ell-1$, assume that $\hat{v}_{h}^{(k)}$ is Lipschitz in space with $\left[\hat{v}_{h}^{(k)}(t, .)\right]_{\text {Lip }} \leq\left(\sum_{j=0}^{k-1} e^{j(T+\delta) \frac{\sigma^{2}}{2}}\right) e^{\frac{\sigma^{2}}{2} T}[\phi]_{\text {Lip }}$ and let us prove that $\hat{v}_{h}^{(k+1)}$ is also. By the Lipschitz assumption of $\phi$ we have that for $t \in[T-h, T], \hat{v}_{h}^{(k+1)}(t,$.$) is Lipschitz with$ $\left[\hat{v}_{h}^{(k+1)}(t, .)\right]_{\text {Lip }}=[\phi]_{\text {Lip }}$.

For $t \in[T-2 h, T-h)$, we have that

$$
\begin{aligned}
& \left|\hat{v}_{h}^{(k+1)}(t, x)-\hat{v}_{h}^{(k+1)}(t, y)\right| \\
\leq & \max \left(e^{-r h} E\left[\left|\hat{v}_{h}^{(k+1)}\left(t+h, P_{R}\left(\hat{X}_{t+h}^{t, x}\right)\right)-\hat{v}_{h}^{(k+1)}\left(t+h, P_{R}\left(\hat{X}_{t+h}^{t, y}\right)\right)\right|\right]\right. \\
& \left.;|\phi(x)-\phi(y)|+e^{-r \delta} E\left[\left|\hat{v}_{h}^{(k)}\left(t+\delta, P_{R}\left(\hat{X}_{t+\delta}^{t, x}\right)\right)-\hat{v}_{h}^{(k)}\left(t+\delta, P_{R}\left(\hat{X}_{t+\delta}^{t, y}\right)\right)\right|\right]\right) \\
\leq & \max \left(e^{-r h}[\phi]_{\text {Lip }} E\left[\left|\hat{X}_{t+h}^{t, x}-\hat{X}_{t+h}^{t, y}\right|\right] ;[\phi]_{\text {Lip }}|x-y|\left(1+\sum_{j=1}^{k} e^{j(T+\delta) \frac{\sigma^{2}}{2}}\right)\right) \\
\leq & \sum_{j=0}^{k} e^{j(T+\delta) \frac{\sigma^{2}}{2}}[\phi]_{\text {Lip }}|x-y| .
\end{aligned}
$$

By repeating the same reasoning for $t$ on each interval of the form $[T-(i+1) h, T-i h)$, for $i=2, \ldots, M-1$, we obtain that $\hat{v}_{h}^{(k+1)}(t,$.$) is Lipschitz in space with$ $\left[\hat{v}_{h}^{(k+1)}(t, .)\right]_{\text {Lip }} \leq e^{\frac{\sigma^{2}}{2}(i-1) h} \sum_{j=0}^{k} e^{j(T+\delta) \frac{\sigma^{2}}{2}}[\phi]_{\text {Lip }}|x-y|$.

We deduce then the desired result.

\subsection{Convergence of the scheme}

We focus now on the convergence (when $h$ goes to zero) of the solution $\hat{v}_{h}^{(k)}$ of (4.7)-(4.8) toward the value function $v^{(k)}$ solution of (4.2)-(4.3). Following Barles and Souganidis [3], we must show that the scheme $S^{h, N, R, k}$ defined by (4.9) is monotone, stable and consistent. We will prove such properties by induction arguments. For the consistency at step $k+1$ $(k \geq 1)$, we need the convergence of the numerical scheme at step $k$. Let us first define the monotonicity, stability and consistency properties.

Definition 4.1 For $k=1, \ldots, \ell$, we say that $S^{h, N, R, k}$ defined by (4.9) is monotonous if

$$
S^{h, N, R, k}\left(t, x, p_{k}, a_{k}, a_{k-1}\right) \geq S^{h, N, R, k}\left(t, x, p_{k}, b_{k}, a_{k-1}\right)
$$


if $a_{k} \leq b_{k}$ for all $(t, x) \in[0, T] \times(0, \infty), p_{k} \in \mathbb{R}, a_{k}, b_{k}$ and $a_{k-1}$ Lipschitz in space and satisfying the linear growth condition.

Definition 4.2 For $k=1, \ldots, \ell$, we say that $S^{h, N, R, k}$ is stable if for all $h>0$, there exists a solution $\hat{v}_{h}^{(k)}$ of (4.7)-(4.8) which is uniformly bounded in $h, N$ and $R$.

Definition 4.3 For $k=1, \ldots, \ell$, we say that $S^{h, N, R, k}$ is consistent if for all Lipschitz function $\psi \in \mathcal{C}^{1,2}([0, T] \times(0, \infty))$, and all $(t, x) \in[0, T] \times(0, \infty)$

$$
\begin{aligned}
& \lim _{\substack{N \sqrt{h}, R \rightarrow \infty \\
h \rightarrow 0,(s, y) \rightarrow(t, x)}} \min \left\{\frac{\psi(s, y)-e^{-r h} E\left[\psi\left(s+h, P_{R}\left(\hat{X}_{s+h}^{s, y}\right)\right)\right]}{h} ; \psi(s, y)-\phi_{\hat{v}_{h}^{(k-1)}}^{N, R}(s, y)\right\} \\
& =\min \left\{-\frac{\partial \psi}{\partial t}(t, x)-\mathcal{L} \psi(t, x) ; \psi(t, x)-\phi_{v^{(k-1)}}(t, x)\right\},
\end{aligned}
$$

where $\hat{v}_{h}^{(k-1)}$ is the solution of (4.7)-(4.8), $v^{(k-1)}$ is the viscosity solution of (4.2)-(4.3), and

$$
\mathcal{L} \psi(t, x):=-r \psi(t, x)+\frac{1}{2} \sigma^{2} x^{2} \frac{\partial^{2} \psi}{\partial x^{2}}(t, x)+r x \frac{\partial \psi}{\partial x}(t, x)
$$

Let us now present and prove the convergence theorem.

Theorem 4.1 Assume that for $k=1, \ldots, \ell, S^{h, N, R, k}$ is monotonous, consistent and stable for $(t, x) \in D$, where $D$ is a subset of $[0, T] \times(0, \infty)$. Then, as $h \rightarrow 0, N \sqrt{h}$ and $R \rightarrow \infty$, the solution $\hat{v}_{h}^{(k)}$ of (4.7)-(4.8) converges locally uniformly in $D$ to the unique viscosity solution $v^{(k)}$ of (4.2)-(4.3).

Proof. For $k \in\{1, \ldots, \ell\}$, we define

$$
\begin{aligned}
& \bar{v}^{(k)}(t, x):=\limsup _{\substack{N \sqrt{h}, R \rightarrow \infty \\
(s, y) \rightarrow(t, x), h \rightarrow 0}} \hat{v}_{h}^{(k)}(s, y), \quad \underline{v}^{(k)}(t, x):=\liminf _{\substack{N \sqrt{h}, R \rightarrow \infty \\
(s, y) \rightarrow(t, x), h \rightarrow 0}} \hat{v}_{h}^{(k)}(s, y) \\
& \text { and } \bar{v}^{(k)}(T, x):=\underline{v}^{(k)}(T, x):=\phi(x) .
\end{aligned}
$$

We claim that $\bar{v}^{(k)}$ and $\underline{v}^{(k)}$ are respectively sub- and super-solutions of (4.2)-(4.3). Assume for the moment that this claim is true; then, by the comparison theorem (see [4]) we obtain that $\bar{v}^{(k)} \leq \underline{v}^{(k)}$ on $\mathrm{D}$. But the opposite inequality is obvious by the definition of $\bar{v}^{(k)}$ and $\underline{v}^{(k)}$, hence $v^{(k)}=\bar{v}^{(k)}=\underline{v}^{(k)}$ is the unique continuous solution of (4.2)-(4.3). This fact together with (4.12) also imply the local uniform convergence of $\hat{v}_{h}^{(k)}$ to $v^{(k)}$.

Our aim now is to prove the above claim.

Let us prove that $\bar{v}^{(k)}$ is a viscosity sub-solution of the HJBVI (4.2)-(4.3). Let $\left(t_{0}, x_{0}\right)$ be a strict local maximum of $\bar{v}^{(k)}-\psi$ on $D$ such that

$\forall(t, x) \in B\left(\left(t_{0}, x_{0}\right), r\right) \backslash\left\{\left(t_{0}, x_{0}\right)\right\} \cap D$

$$
\bar{v}^{(k)}(t, x)-\psi(t, x)<\bar{v}^{(k)}\left(t_{0}, x_{0}\right)-\psi\left(t_{0}, x_{0}\right):=0,
$$


where $\psi$ is bounded and infinitely differentiable function defined on $D$, such that

$$
\begin{aligned}
& \psi \geq 2 C \quad \text { out side the ball } B\left(\left(t_{0}, x_{0}\right), r\right) \\
& \psi \leq C \quad \text { on the ball } B\left(\left(t_{0}, x_{0}\right), r\right) .
\end{aligned}
$$

Where $C>0$ is such that $\left|\hat{v}_{h}^{(k)}(t, x)\right| \leq C, \forall(t, x) \in D, h, R>0$ and $N \in \mathbb{N} \backslash\{0\}$, the existence of $C$ is insured by the boundedness of the function $\phi$.

It is known that there exist sequences $h_{n}>0, N_{n}$ in $\mathbb{N} \backslash\{0\}, R_{n}$ in $[0, \infty)$ and $\left(s_{n}, y_{n}\right) \in D$ such that as $n \rightarrow \infty, h_{n} \rightarrow 0, R_{n}, N_{n} \sqrt{h_{n}} \rightarrow \infty,\left(s_{n}, y_{n}\right) \rightarrow\left(t_{0}, x_{0}\right), \hat{v}_{h_{n}}^{(k)}\left(s_{n}, y_{n}\right) \rightarrow$ $\bar{v}^{(k)}\left(t_{0}, x_{0}\right)$ and $\left(s_{n}, y_{n}\right)$ is a global maximum point of $\hat{v}_{h_{n}}^{(k)}-\psi$. Let us prove that

$$
\min \left\{-\frac{\partial \psi}{\partial t}\left(t_{0}, x_{0}\right)-\mathcal{L} \psi\left(t_{0}, x_{0}\right) ; \psi\left(t_{0}, x_{0}\right)-\phi_{v^{(k-1)}}\left(t_{0}, x_{0}\right)\right\} \leq 0 .
$$

Denoting by $\rho_{n}$ the quantity $\hat{v}_{h_{n}}^{(k)}\left(s_{n}, y_{n}\right)-\psi\left(s_{n}, y_{n}\right)$, then we have $\rho_{n} \rightarrow 0$ and

$$
\hat{v}_{h_{n}}^{(k)}(t, x) \leq \psi(t, x)+\rho_{n} \text { for all }(t, x) \in D .
$$

The definition of $\hat{v}_{h_{n}}^{(k)}$, the monotonicity of $S^{h_{n}, N_{n}, R_{n}, k}$ and (4.15) yield

$$
\begin{aligned}
& S^{h_{n}, N_{n}, R_{n}, k}\left(s_{n}, y_{n}, \psi\left(s_{n}, y_{n}\right)+\rho_{n}, \psi+\rho_{n}, v_{h_{n}}^{(k-1)}\right) \\
& \leq S^{h_{n}, N_{n}, R_{n}, k}\left(s_{n}, y_{n}, \psi\left(s_{n}, y_{n}\right)+\rho_{n}, \hat{v}_{h_{n}}^{(k)}, v_{h_{n}}^{(k-1)}\right)=0 .
\end{aligned}
$$

Taking limits in (4.16) and using the consistency of $S^{h_{n}, N_{n}, R_{n}, k}$ we obtain

$$
\begin{aligned}
0 & \geq \lim _{n} S^{h_{n}, N_{n}, R_{n}, k}\left(s_{n}, y_{n}, \psi\left(s_{n}, y_{n}\right)+\rho_{n}, \psi+\rho_{n}, v_{h_{n}}^{(k-1)}\right) \\
& \geq \liminf _{\substack{N \sqrt{h}, R \rightarrow \infty, \rho \rightarrow 0 \\
h \rightarrow 0,(s, y) \rightarrow\left(t_{0}, x_{0}\right)}} S^{h, N, R, k}\left(s, y, \psi(s, y)+\rho, \psi+\rho, v_{h_{n}}^{(k-1)}\right) \\
& \geq \min \left\{-\frac{\partial \psi}{\partial t}\left(t_{0}, x_{0}\right)-\mathcal{L} \psi\left(t_{0}, x_{0}\right) ; \psi\left(t_{0}, x_{0}\right)-\phi_{v^{(k-1)}}\left(t_{0}, x_{0}\right)\right\},
\end{aligned}
$$

then inequality (4.14) is proved and we conclude then that $\bar{v}^{(k)}$ is a viscosity sub-solution of the HJBVI (4.2)-(4.3). We can prove identically that $\underline{v}^{(k)}$ is a viscosity super-solution of the HJBVI (4.2)-(4.3). Then by the comparison theorem we obtain that $\hat{v}_{h}^{(k)}$ converges locally uniformly to the unique viscosity solution of (4.2)-(4.3).

Let us now prove the monotonicity, stability and consistency of our numerical scheme. For the consistency property, we will prove that our scheme is consistent for $(t, x) \in$ $[0, T] \backslash\{T-\delta\} \times(0, \infty)$. Then from the last theorem we obtain the local uniform convergence of $\hat{v}_{h}^{(k)}$ to $v^{(k)}$ in $[0, T] \backslash\{T-\delta\} \times(0, \infty)$.

The monotonicity of the scheme is obvious.

Proposition 4.2 For all $k=1, \ldots, \ell, S^{h, N, R, k}$ is monotonous.

Proposition 4.3 For $k=1, \ldots, \ell, S^{h, N, R, k}$ is stable. 
Proof. We have that for all $(t, x) \in[0, T-h) \times(0, \infty)$

$$
\begin{aligned}
& S^{h, N, R, k}\left(t, x, \hat{v}_{h}^{(k)}(t, x), \hat{v}_{h}^{(k)}, \hat{v}_{h}^{(k-1)}\right)=0 \Leftrightarrow \\
& \hat{v}_{h}^{(k)}(t, x)=\max \left(e^{-r h} E\left[\hat{v}_{h}^{(k)}\left(t+h, P_{R}\left(\hat{X}_{t+h}^{t, x}\right)\right)\right] ; \phi_{\hat{v}_{h}^{N-1)}}^{(k-R}(t, x)\right) .
\end{aligned}
$$

Our aim now is to prove that for $k=1, \ldots, \ell, \hat{v}_{h}^{(k)}$ is uniformly bounded in $h, N$ and $R$. We define the pay-off function $\phi(x):=(K-x)_{+}$, where $K>0$.

For $k=1$ :

For $t \in[T-h, T], \quad \hat{v}_{h}^{(1)}(t, x)=\phi(x) \leq K$.

For $t \in[T-2 h, T-h)$,

$$
\begin{aligned}
\hat{v}_{h}^{(1)}(t, x) & =\max \left(e^{-r h} E\left[\hat{v}_{h}^{(1)}\left(t+h, P_{R}\left(\hat{X}_{t+h}^{t, x}\right)\right)\right] ; \phi(x)\right) \\
& \leq K
\end{aligned}
$$

where the last inequality is deduced from the fact that $t+h \in[T-h, T)$. Then $\hat{v}_{h}^{(1)}$ is uniformly bounded in $h, N$ and $R$ when $t \in[T-2 h, T-h)$. By repeating this reasoning on each interval of the form $[T-(i+1) h, T-i h)$, for $i=2, \ldots, M-1$, we obtain that $\hat{v}_{h}^{(1)}$ is uniformly bounded in $h, N$ and $R$.

Let $1 \leq k \leq \ell$, assume that $\hat{v}_{h}^{(k)}$ is uniformly bounded in $h, N$ and $R$ and let us prove that $\hat{v}_{h}^{(k)}$ is also.

For $t \in[T-h, T], \quad \hat{v}_{h}^{(k+1)}(t, x)=\phi(x) \leq K$.

For $t \in[T-2 h, T-h)$ there are two cases:

$1^{\text {st }}$ case: If $t \in(T-\delta, T]$,

$$
\begin{aligned}
\hat{v}_{h}^{(k+1)}(t, x) & =\max \left(e^{-r h} E\left[\hat{v}_{h}^{(k+1)}\left(t+h, P_{R}\left(\hat{X}_{t+h}^{t, x}\right)\right)\right] ; \phi(x)\right) \\
& \leq K
\end{aligned}
$$

$2^{\text {nd }}$ case: If $t \in[0, T-\delta]$,

$$
\hat{v}_{h}^{(k+1)}(t, x)=\max \left(e^{-r h} E\left[\hat{v}_{h}^{(k+1)}\left(t+h, P_{R}\left(\hat{X}_{t+h}^{t, x}\right)\right)\right] ; \phi(x)+e^{-r \delta} E\left[\hat{v}_{h}^{(k)}\left(t+\delta, X_{t+\delta}^{t, x}\right)\right]\right) .
$$

We have that when $t \in[T-h, T], \hat{v}_{h}^{(k+1)}$ is uniformly bounded in $h, N$ and $R$, then by the induction assumption and the definition of $\hat{v}_{h}^{(k+1)}$, we deduce that it is uniformly bounded in $h, N$ and $R$.

We repeat the same argument on each interval of the form $[T-(i+1) h, T-i h)$, for $i=2, \ldots, M-1$ we obtain that $\hat{v}_{h}^{(k+1)}$ is uniformly bounded in $h, N$ and $R$. We conclude then the stability of $S^{h, N, R, k}$ for $k=1, \ldots, \ell$.

The definition of $\phi_{\hat{v}_{h}^{(k)}}^{N, R}$ differs if $t \leq T-\delta$ or $t>T-\delta$. Then we will prove by induction the consistency of the numerical scheme for all $t \in[0, T] \backslash\{T-\delta\}$, here we choose $D=[0, T] \backslash\{T-\delta\} \times(0, \infty)$. We need the following two Lemmas. 
Lemma 4.1 For all Lipschitz function $\psi \in \mathcal{C}^{1,2}([0, T] \times(0, \infty)), \forall(s, y) \in[0, T] \times(0, \infty)$ and for $N=h^{\alpha}$, where $\alpha<-1 / 2$, we have

$$
\lim _{\substack{R \rightarrow \infty \\ h \rightarrow 0}} \frac{\left|E\left[\psi\left(s+h, P_{R}\left(\hat{X}_{s+h}^{s, y}\right)\right)\right]-E\left[\psi\left(s+h, X_{s+h}^{s, y}\right)\right]\right|}{h}=0
$$

Proof. We have that for all Lipschitz function $\psi \in \mathcal{C}^{1,2}([0, T] \times(0, \infty))$ and for all $(s, y) \in[0, T] \times(0, \infty)$

$$
\left|E\left[\psi\left(s+h, P_{R}\left(\hat{X}_{s+h}^{s, y}\right)\right)\right]-E\left[\psi\left(s+h, X_{s+h}^{s, y}\right)\right]\right| \leq C E\left[\left|P_{R}\left(\hat{X}_{s+h}^{s, y}\right)-X_{s+h}^{s, y}\right|\right] .
$$

So by applying the dominated convergence theorem we obtain that

$$
\lim _{R \rightarrow \infty}\left|E\left[\psi\left(s+h, P_{R}\left(\hat{X}_{s+h}^{s, y}\right)\right)\right]-E\left[\psi\left(s+h, X_{s+h}^{s, y}\right)\right]\right| \leq C E\left[\left|\hat{X}_{s+h}^{s, y}-X_{s+h}^{s, y}\right|\right] .
$$

It is well known that there exists a normal random variable $U$ such that, $\forall u \in[s, T]$ $X_{u}^{s, y}=y e^{\left(r-\frac{\sigma^{2}}{2}\right)(u-s)+\sigma \sqrt{u-s} U}$ a.s., then

$$
\begin{aligned}
E\left[\left|\hat{X}_{s+h}^{s, y}-X_{s+h}^{s, y}\right|\right] & =y e^{\left(r-\frac{\sigma^{2}}{2}\right) h} E\left[\left|e^{\sigma \sqrt{h} \hat{U}}-e^{\sigma \sqrt{h} U}\right|\right] \\
& \leq C y \sigma \sqrt{h} e^{\left(r-\frac{\sigma^{2}}{2}\right) h} E\left[\left(e^{\sigma \sqrt{h} \hat{U}}+e^{\sigma \sqrt{h} U}\right)|\hat{U}-U|\right] \\
& \leq C y \sigma \sqrt{h} e^{\left(r-\frac{\sigma^{2}}{2}\right) h} \sqrt{E\left[\left(e^{\sigma \sqrt{h} \hat{U}}+e^{\sigma \sqrt{h} U}\right)^{2}\right]} \sqrt{E\left[|\hat{U}-U|^{2}\right]} \\
& \leq C y \sigma \sqrt{h} e^{\left(r-\frac{\sigma^{2}}{2}\right) h}\left(\sqrt{E\left[e^{2 \sigma \sqrt{h} \hat{U}}\right]}+\sqrt{E\left[e^{2 \sigma \sqrt{h} U}\right]}\right) \sqrt{E\left[|\hat{U}-U|^{2}\right]} .
\end{aligned}
$$

As in (4.11), we have $E\left[e^{2 \sigma \sqrt{h} \hat{U}}\right] \leq e^{2 \sigma^{2} h}$, and by Zador's Theorem we have $\sqrt{E\left[|\hat{U}-U|^{2}\right]}=$ $O\left(\frac{1}{N}\right)$, then

$$
E\left[\left|\hat{X}_{s+h}^{s, y}-X_{s+h}^{s, y}\right|\right] \leq \frac{1}{N} C y \sigma \sqrt{h} e^{\left(r+\frac{\sigma^{2}}{2}\right) h}
$$

Let $N=h^{\alpha}$, where $\alpha<-\frac{1}{2}$, then

$$
\frac{E\left[\left|\hat{X}_{s+h}^{s, y}-X_{s+h}^{s, y}\right|\right]}{h} \leq \varepsilon(h),
$$

where $\varepsilon(h):=C y \sigma h^{-\frac{1}{2}-\alpha}$ goes to 0 when $h$ goes to 0 . We deduce then by inequalities (4.19) and (4.21) that

$$
\frac{\left|E\left[\psi\left(s+h, P_{R}\left(\hat{X}_{s+h}^{s, y}\right)\right)\right]-E\left[\psi\left(s+h, X_{s+h}^{s, y}\right)\right]\right|}{h} \rightarrow 0 \text {, when } h \rightarrow 0 \text { and } R \rightarrow \infty \text {. }
$$


Lemma 4.2 For all Lipschitz function $\psi \in \mathcal{C}^{1,2}([0, T] \times(0, \infty))$ and for all $(t, x) \in[0, T] \times(0, \infty)$, we have

$$
\lim _{\substack{h \rightarrow 0 \\(s, y) \rightarrow(t, x)}} \frac{\psi(s, y)-e^{-r h} E\left[\psi\left(s+h, X_{s+h}^{s, y}\right)\right]}{h}=-\frac{\partial \psi}{\partial t}(t, x)-\mathcal{L} \psi(t, x) .
$$

Proof. By applying Itô's formula to $u \rightarrow e^{-r(u-s)} \psi\left(u, X_{u}^{s, y}\right)$ in $[s, s+h]$ we obtain

$$
\begin{aligned}
e^{-r h} \psi\left(s+h, X_{s+h}^{s, y}\right)-\psi(s, y)= & \int_{s}^{s+h} e^{-r(u-s)}\left(\frac{\partial \psi}{\partial t}\left(u, X_{u}^{s, y}\right)+\mathcal{L} \psi\left(u, X_{u}^{s, y}\right)\right) d u \\
& +\int_{s}^{s+h} e^{-r(u-s)} \frac{\partial \psi}{\partial x}\left(u, X_{u}^{s, y}\right) \sigma X_{u}^{s, y} d W_{u} .
\end{aligned}
$$

By dividing the last inequality by $h$ and taking the expectation we obtain

$\frac{e^{-r h} E\left[\psi\left(s+h, X_{s+h}^{s, y}\right)\right]-\psi(s, y)}{h}=\frac{1}{h} E\left[\int_{s}^{s+h} e^{-r(u-s)}\left(\frac{\partial \psi}{\partial t}\left(u, X_{u}^{s, y}\right)+\mathcal{L} \psi\left(u, X_{u}^{s, y}\right)\right) d u\right]$.

By sending $h$ to 0 and using the dominated convergence theorem we obtain that

$$
\lim _{h \rightarrow 0} \frac{\psi(s, y)-e^{-r h} E\left[\psi\left(s+h, X_{s+h}^{s, y}\right)\right]}{h}=-\frac{\partial \psi}{\partial t}(s, y)-\mathcal{L} \psi(s, y) .
$$

Then by the regularity of $\psi$ we obtain equality (4.23).

Proposition 4.4 For $k=1, \ldots, \ell, S^{h, N, R, k}$ is consistent for $N=h^{\alpha}$, where $\alpha<-1 / 2$, and for $t \in[0, T] \backslash\{T-\delta\}$.

Proof. By Lemma 4.1 and Lemma 4.2 we obtain that for all Lipschitz function $\psi \in \mathcal{C}^{1,2}([0, T] \times(0, \infty))$, and all $(t, x) \in[0, T] \times(0, \infty)$

$$
\lim _{\substack{R \rightarrow \infty \\ h \rightarrow 0,(s, y) \rightarrow(t, x)}} \frac{\psi(s, y)-e^{-r h} E\left[\psi\left(s+h, P_{R}\left(\hat{X}_{s+h}^{s, y}\right)\right)\right]}{h}=-\frac{\partial \psi}{\partial t}(t, x)-\mathcal{L} \psi(t, x) .
$$

Then to prove the consistency of $S^{h, N, R, k+1}$ we will just prove that

$$
\lim _{\substack{N \sqrt{h}, R \rightarrow \infty \\ h \rightarrow 0,(s, y) \rightarrow(t, x)}} \phi_{\hat{v}_{h}^{(k)}}^{N, R}(s, y)=\phi_{v^{(k)}}(t, x) .
$$

We will prove this equality by induction.

For $k=1$, by the assumption $v_{h}^{(0)} \equiv \hat{v}_{h}^{(0)} \equiv 0$, we obtain that $\phi_{v^{(0)}}(t, x)=\phi(x)$ and $\phi_{\hat{v}_{h}^{(0)}}^{N, R}(s, y)=\phi(y)$, then equality (4.26) is satisfied for $k=1$.

Let $1 \leq k \leq \ell-1$, assume that equality (4.26) is satisfied for $k$, then $S^{h, N, R, k}$ is consistent, and let us prove that so is for $k+1$. We have that $S^{h, N, R, k}$ is monotonous, stable and consistent for $t \neq T-\delta$, then by theorem 4.1, we obtain that for $t \neq T-\delta, \hat{v}_{h}^{(k)}$ converges to $v^{(k)}$. 
For $s$ near $t$ we have two cases: $s$ and $t$ are in $[0, T-\delta)$ or in $(T-\delta, T]$. $1^{\text {st }}$ case: For $s$ and $t$ in $[0, T-\delta)$

$$
\begin{aligned}
\left|\phi_{\hat{v}_{h}^{(k)}}^{N, R}(s, y)-\phi_{v^{(k)}}(t, x)\right| & \leq|\phi(y)-\phi(x)|+E\left[\left|\hat{v}_{h}^{(k)}\left(s+\delta, P_{R}\left(\hat{X}_{s+\delta}^{s, y}\right)\right)-v^{(k)}\left(t+\delta, X_{t+\delta}^{t, x}\right)\right|\right] \\
& \leq C|x-y|+E\left[\left|\hat{v}_{h}^{(k)}\left(s+\delta, P_{R}\left(\hat{X}_{s+\delta}^{s, y}\right)\right)-v^{(k)}\left(s+\delta, X_{s+\delta}^{s, y}\right)\right|\right] \\
& +E\left[\left|v^{(k)}\left(s+\delta, X_{s+\delta}^{s, y}\right)-v^{(k)}\left(t+\delta, X_{t+\delta}^{t, x}\right)\right|\right] .
\end{aligned}
$$

Using the Lipschitz property of $\hat{v}_{h}^{(k)}$ and inequality (4.20) we obtain that

$$
\begin{aligned}
E\left[\left|\hat{v}_{h}^{(k)}\left(s+\delta, P_{R}\left(\hat{X}_{s+\delta}^{s, y}\right)\right)-v^{(k)}\left(s+\delta, X_{s+\delta}^{s, y}\right)\right|\right] \leq & E\left[\left|\hat{v}_{h}^{(k)}\left(s+\delta, P_{R}\left(\hat{X}_{s+\delta}^{s, y}\right)\right)-\hat{v}_{h}^{(k)}\left(s+\delta, X_{s+\delta}^{s, y}\right)\right|\right] \\
& +E\left[\left|\hat{v}_{h}^{(k)}\left(s+\delta, X_{s+\delta}^{s, y}\right)-v^{(k)}\left(s+\delta, X_{s+\delta}^{s, y}\right)\right|\right] \\
\leq & \frac{1}{N} C y \sigma \sqrt{h} e^{\left(r+\frac{\sigma^{2}}{2}\right) h} \\
& +E\left[\left|\hat{v}_{h}^{(k)}\left(s+\delta, X_{s+\delta}^{s, y}\right)-v^{(k)}\left(s+\delta, X_{s+\delta}^{s, y}\right)\right|\right] .
\end{aligned}
$$

So by the convergence of $\hat{v}_{h}^{(k)}$ to $v^{(k)}$ we obtain that

$$
\begin{aligned}
& \lim _{\substack{N \sqrt{h}, R \rightarrow \infty \\
h \rightarrow 0,(s, y) \rightarrow(t, x)}} E\left[\left|\hat{v}_{h}^{(k)}\left(s+\delta, P_{R}\left(\hat{X}_{s+\delta}^{s, y}\right)\right)-v^{(k)}\left(s+\delta, X_{s+\delta}^{s, y}\right)\right|\right]=0 . \\
& E\left[\left|v^{(k)}\left(s+\delta, X_{s+\delta}^{s, y}\right)-v^{(k)}\left(t+\delta, X_{t+\delta}^{t, x}\right)\right|\right] \leq E\left[\left|v^{(k)}\left(s+\delta, X_{s+\delta}^{s, y}\right)-v^{(k)}\left(s+\delta, X_{t+\delta}^{t, x}\right)\right|\right] \\
&+E\left[\left|v^{(k)}\left(s+\delta, X_{t+\delta}^{t, x}\right)-v^{(k)}\left(t+\delta, X_{t+\delta}^{t, x}\right)\right|\right] \\
& \leq C E\left[\left|X_{s+\delta}^{s, y}-X_{t+\delta}^{t, x}\right|\right]+C E\left[1+\left|X_{t+\delta}^{t, x}\right|\right] \sqrt{|s-t|} \\
& \leq C|x-y|+C(1+|x|) \sqrt{|s-t|},
\end{aligned}
$$

where the second inequality is deduced by the continuity of $v^{(k)}$, see [4], and the last one is deduced by Pham [16]. We conclude then that

$$
\lim _{\substack{N \sqrt{h}, R \rightarrow \infty \\ h \rightarrow 0,(s, y) \rightarrow(t, x)}} E\left[\left|v_{h}^{(k)}\left(s+\delta, \hat{X}_{s+\delta}^{s, y}\right)-v^{(k)}\left(t+\delta, X_{t+\delta}^{t, x}\right)\right|\right]=0 .
$$

$2^{\text {nd }}$ case: For $s$ and $t$ in $(T-\delta, T]$

$$
\left|\phi_{\hat{v}_{h}^{(k)}}^{N, R}(s, y)-\phi_{v^{(k)}}(t, x)\right|=|\phi(y)-\phi(x)| \leq C|x-y|,
$$

which goes to 0 when $(s, y)$ goes to $(t, x)$.

In the two cases we obtain equality (4.26). We deduce then the consistency of $S^{h, N, R, k}$, for $k=1, \ldots, \ell$. 
We have proved that our scheme is monotonous and stable for $t \in[0, T]$ and consistent for $t \in[0, T] \backslash\{T-\delta\}$, then by theorem 4.1 we conclude that $\hat{v}_{h}^{(k)}$ converges locally uniformly on $[0, T] \backslash\{T-\delta\} \times(0, \infty)$ to the unique viscosity solution $v^{(k)}$ of (4.2)-(4.3). By the following corollary we obtain the convergence of $\hat{v}_{h}^{(k)}$, on $[0, T] \times(0, \infty)$, to the unique viscosity solution $v^{(k)}$ of (4.2)-(4.3).

Corollary 4.1 If $\hat{v}_{h}^{(k)}$ converges locally uniformly on $[0, T] \backslash\{T-\delta\} \times(0, \infty)$ to the unique viscosity solution $v^{(k)}$ of (4.2)-(4.3). Then $\hat{v}_{h}^{(k)}$ converges on $[0, T] \times(0, \infty)$ to the unique viscosity solution $v^{(k)}$ of (4.2)-(4.3).

Proof. We have that $\hat{v}_{h}^{(k)}$ converges locally uniformly to $v^{(k)}(t, x)$ in $[0, T] \backslash\{T-\delta\} \times(0, \infty)$ then $\forall x \in(0, \infty)$

$$
\lim _{\substack{(s, y) \rightarrow(T-\delta, x) \\ s<T-\delta}} \hat{v}_{h}^{(k)}(s, y)=v^{(k)}\left((T-\delta)^{-}, x\right)
$$

and

$$
\lim _{\substack{(s, y) \rightarrow(T-\delta, x) \\ s>T-\delta}} \hat{v}_{h}^{(k)}(s, y)=v^{(k)}\left((T-\delta)^{+}, x\right)
$$

Equalities (4.30) and (4.31) added to the continuity of $v^{(k)}$ on $[0, T] \times(0, \infty)$, imply the convergence of $\hat{v}_{h}^{(k)}$ to $v^{(k)}$ on $(T-\delta, x)$.

We conclude then that $\hat{v}_{h}^{(k)}$ converges on $[0, T] \times(0, \infty)$ to the unique viscosity solution $v^{(k)}$ of (4.2)-(4.3).

Note that the approximation scheme is not yet fully implementable, since it requires an approximation method for the expectations arising in (4.9). This is the concern of the next section.

\section{$5 \quad$ Numerical solution for one-dimensional case}

In this section, we introduce an implementable scheme. We discretize in time the optimal stopping problem (4.5). Let $M \in \mathbb{N} \backslash\{0\}$ and $h=T / M$ be the time step. We assume that there exists $i_{0} \in\{1, \ldots, M\}$ such that $\delta=i_{0} h$. For $q=0, \ldots, M$, we denote by $t_{q}=q h$. We define for $i=1, \ldots, N, q=M, \ldots, 0, x_{i}^{q}:=x e^{\left(r-\frac{\sigma^{2}}{2}\right) q h+\sigma \sqrt{q h} u_{i}}, x_{i}^{0}:=x$, $x^{q}:=\left\{x_{1}^{q}, \ldots, x_{N}^{q}\right\}$, where $\left(u_{1}, \ldots, u_{N}\right)$ is an optimal quantization grid of the standard univariate normal distribution.

Our aim now is to calculate the value $\hat{v}_{h}^{(k)}(0, x)$ for $x>0$. 
We define $\hat{V}_{t, x}^{(k)}$ solution of

$$
\left\{\begin{aligned}
& \hat{V}_{t, x}^{(k)}\left(t_{M}, x_{i}^{M}\right)=\phi\left(x_{i}^{M}\right) \quad \forall i=1, \ldots, N \\
& \hat{V}_{t, x}^{(k)}\left(t_{q}, x_{i}^{q}\right)=\max \left\{\quad e^{-r h} \sum_{j=1}^{N} \hat{V}_{t, x}^{(k)}\left(t_{q+1}, P_{R}\left(x_{j}^{q+1}\right)\right) \frac{\mathbb{P}\left(X_{q+1}^{t, x} \in C_{j}\left(x^{q+1}\right), X_{q}^{t, x} \in C_{i}\left(x^{q}\right)\right)}{\mathbb{P}\left(X_{q}^{t, x} \in C_{i}\left(x^{q}\right)\right)},\right. \\
&\left.\hat{\phi}_{h, t, x}^{(k)}\left(t_{q}, x_{i}^{q}\right)\right\} \quad \forall i=1, \ldots, N, q=M-1, \ldots, 0,
\end{aligned}\right.
$$

where

$$
\begin{aligned}
\hat{\phi}_{h, t, x}^{(k)}\left(t_{q}, x_{i}^{q}\right)= & \phi\left(x_{i}^{q}\right)+e^{-r \delta} \\
& \sum_{j=1}^{N} \hat{V}_{t, x}^{(k-1)}\left(t_{q+i_{0}}, x_{j}^{q+i_{0}}\right) \frac{\mathbb{P}\left(X_{q+i_{0}}^{t, x} \in C_{j}\left(x^{q+i_{0}}\right), X_{q}^{t, x} \in C_{i}\left(x^{q}\right)\right)}{\mathbb{P}\left(X_{q}^{t, x} \in C_{i}\left(x^{q}\right)\right)} \quad \forall q=0, \ldots, M-i_{0}, \\
\hat{\phi}_{h, t, x}^{(k)}\left(t_{q}, x_{i}^{q}\right)= & \phi\left(x_{i}^{q}\right) \quad \forall q=M-i_{0}+1, \ldots, M .
\end{aligned}
$$

The following table presents Swing put option values for different numbers of simulations for $\ell=5, T=1$ year, $\delta=0.1$ year, $x=K=100, r=0.05, \sigma=0.3, M=50, N=150$ and 250 .

\begin{tabular}{|c|c|c|}
\hline & $\mathrm{N}=150$ & $\mathrm{~N}=250$ \\
\hline$\hat{V}_{t, x}^{(1)}(0, x)$ & 9.87 & 9.86 \\
\hline$\hat{V}_{t, x}^{(2)}(0, x)$ & 19.21 & 19.34 \\
\hline$\hat{V}_{t, x}^{(3)}(0, x)$ & 27.75 & 28.41 \\
\hline$\hat{V}_{t, x}^{(4)}(0, x)$ & 36.49 & 36.49 \\
\hline$\hat{V}_{t, x}^{(5)}(0, x)$ & 44.25 & 44.26 \\
\hline
\end{tabular}

This result is similar to those of Carmona and Touzi [6].

We have implemented and tested the above algorithm for the swing put option with the following characteristics: maturity $T=1$ year, refraction period $\delta=0.1$ year, $r=0.05$, $\sigma=0.30$, maximal number of exercise rights $\ell=5$ and the number of points in the optimal quantization grid $N=150$. The following figure gives the plots of the graphs of the functions $x \rightarrow v^{(k)}(0, x)$ for $k=1,2, \ldots, 5$.

\subsection{Complexity of the algorithm}

The computation of the value function has not an expensive computation cost. Indeed, this grid contains $O(M)$ points, and at each point $t_{q}, q=0, \ldots, M$, one has to compute (1) the approximation of conditional expectation $E\left[\hat{v}_{h}^{(k)}\left(t_{q}+h, P_{R}\left(\hat{X}_{t_{q}+\delta}^{t, x}\right)\right)\right]$ that costs $O(N)$ unitary operations,

(2) the localization procedure has constant computational cost $O(1)$. 


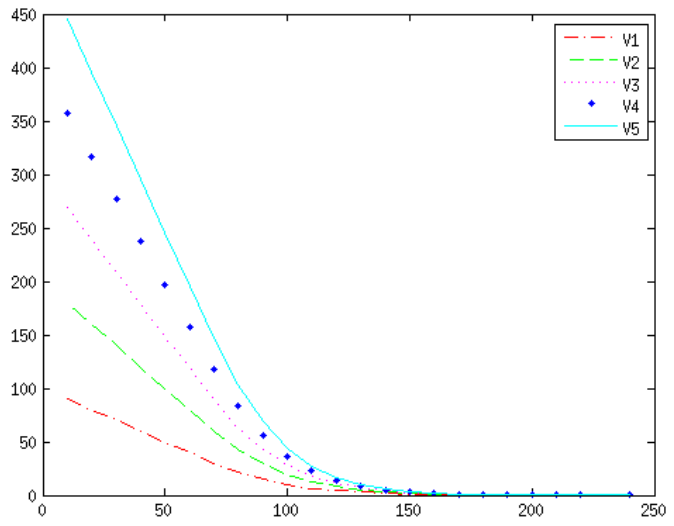

Figure 1: graph of the functions $v^{(k)}(0,$.$) for k=1,2,3,4$ and 5

Therefore, we obtain

$$
\text { complexity }=O(M N) .
$$

Actually, setting $K=\max (M, N)$, the complexity of the algorithm is $O\left(K^{2}\right)$. Yet, practical implementation of the algorithm can achieve much better performance. In the optimal quantization for the computation of the expectations in the numerical algorithm, we can choose $N=O\left(M^{1 / 2+\epsilon}\right)$ for all $\epsilon>0$. In this case, the complexity is reduced to

$$
\text { complexity }=O\left(M^{5 / 2+\epsilon}\right) \text { for all } \epsilon>0,
$$

which is satisfactory when considering that there are $O(M)$ points to compute in the time axis,. If we consider the space discretization with $\bar{M}$ points, the complexity of the algorithm will be

$$
\text { complexity }=O\left(\bar{M} M^{5 / 2+\epsilon}\right) \text { for all } \epsilon>0 .
$$

\subsection{Comparison with the finite-difference scheme}

Let us compare our result with usual finite difference scheme. Let us briefly introduce the class of theta schemes. We refer the reader to Lapeyre et al. [12] for a complete discussion about this class of schemes. We will assume that the value function is sufficiently smooth, and we focus in this section on the diffusive part of the HJBVI, so that the target equation we have to solve is

$$
\frac{\partial v^{(k)}}{\partial t}(t, x)+\mathcal{L} v^{(k)}(t, x)=0 \quad \forall(t, x) \in[0, T) \times(0, \infty),
$$

together with a terminal condition on $\{T\} \times(0, \infty)$, where

$$
\mathcal{L} v^{(k)}(t, x)=-r v^{(k)}(t, x)+\frac{1}{2} \sigma^{2} x^{2} \frac{\partial^{2} v^{(k)}}{\partial x^{2}}(t, x)+r x \frac{\partial v^{(k)}}{\partial x}(t, x) .
$$


To solve numerically this Kolmogorov parabolic equation with finite time horizon, we can discretize it using a theta scheme. Let $\theta \in[0,1], \Delta:=R / M$, with $M \in \mathbb{N}^{*}$, denote the spatial step, and $\mathcal{O}_{\Delta}:=\{i \Delta, i=0, \ldots, M\}$ be the associated spatial grid. We denote by $h:=T / K$, with $K \in \mathbb{N}^{*}$, the time step. The approximation consists in the following:

$$
\frac{\partial v^{(k)}}{\partial t}(t, x)+\mathcal{L} v^{(k)}(t, x) \simeq \mathcal{P}_{h, \Delta}^{\theta} v^{(k)}(t, x),
$$

where

$$
\begin{aligned}
\mathcal{P}_{h, \Delta}^{\theta} v^{(k)}(t, x):= & \frac{v^{(k)}(t+h, x)-v^{(k)}(t, x)}{\Delta t}+\theta L_{\Delta} v^{(k)}(t, x)+(1-\theta) L_{\Delta} v^{(k)}(t+h, x), \\
L_{\Delta} v^{(k)}(t, x):= & \frac{\sigma^{2} x^{2}}{2} \frac{v^{(k)}(t, x+\Delta)-2 v^{(k)}(t, x)+v^{(k)}(t, x-\Delta)}{\Delta^{2}} \\
& +r x \frac{v^{(k)}(t, x+\Delta)-v^{(k)}(t, x-\Delta)}{2 \Delta}-r v^{(k)}(t, x) .
\end{aligned}
$$

The discretized equation is

$$
\mathcal{P}_{h, \Delta}^{\theta} v^{(k)}(t, x)=0 \quad \forall(t, x) \in \mathcal{O}_{\Delta} \times[0, T)
$$

From the finite-differences approximation, we have the following precision:

$$
\frac{\partial v^{(k)}}{\partial t}(t, x)+\mathcal{L} v^{(k)}(t, x)=\mathcal{P}_{h, \Delta}^{\theta} v^{(k)}(t, x)+O\left(h^{p}+\Delta^{q}\right),
$$

where $p$ and $q$ depend on the choice of $\theta$ : if $\theta \neq 1 / 2$ we obtain that $p=1$ and if $\theta=1 / 2$ we obtain that $p=2$ which corresponds to the Crank-Nicholson scheme. Due to the second order derivative in $\mathcal{L}$, and by using standard finite-difference approximation, the rate of convergence for the spatial approximation is $q=2$, for all $\theta \in[0,1]$. Therefore, in our case, we see that theta schemes have order 1 in time and order 2 in space, except for the Crank-Nicholson scheme, which gives an order 2 in time and order 2 in space. For comparison purposes, the optimally quantized scheme that we use has a precision of $o(h)$ in time provided that $N \sim h^{-(1 / 2+\varepsilon)}$, where $N$ is the number of points in the optimal quantization grid. Indeed, we have that

$$
\frac{\partial v^{(k)}}{\partial t}(t, x)+\mathcal{L} v^{(k)}(t, x)=0 \quad \forall(t, x) \in[0, T) \times(0, \infty) .
$$

Then by Lemma 4.1 and Lemma 4.2 we obtain that

$$
\hat{v}_{h}^{(k)}(t, x)=E\left[\hat{v}_{h}^{(k)}\left(t+h, P_{R}\left(\hat{X}_{t+h}^{t, x}\right)\right)\right]+o(h) .
$$

This prompts two comments. First, we see that, in contrast with the finite-difference scheme, the precision of the optimally quantized scheme is controlled by the number of points $N$ of the optimal quantization grid, and not by the space step $\Delta$. Therefore, we can improve the precision by increasing $N$ without increasing the size of the grid, which is very interesting when dealing with high-dimensional state space. Second, using the optimally 
quantized method we keep a precision of $o(h)$. Whereas, using a finite-difference scheme, the above result allows us to choose $M \simeq K^{1 / 4}$, to obtain a precision of $O\left(h^{1 / 2}\right)$ due to spatial approximation. Therefore, by using an optimally quantized scheme, we can obtain a satisfactory precision, while managing efficiently the size of the grid, and subsequently the memory needed to achieve computation, which is highly relevant when dealing with high-dimensional state space.

\section{References}

[1] V. Bally and G. Pagès (2003). A quantization algorithm for solving multidimensional discrete-time optimal stopping problems. Bernoulli 9, 1003-1049.

[2] V. Bally, G. Pagès and J. Printems, (2005). A quantization tree method for pricing and hedging multidimensional American options. Math. Finance 15, 119-168.

[3] G. Barles and P.E. Souganidis (1991). Convergence of approximation schemes for fully nonlinear second order equations, Asymptotic analysis, 4, 271-283.

[4] I. Ben Latifa, J. F. Bonnans and M. Mnif (2013), A general optimal multiple stopping problem with an application to Swing Options, submitted.

[5] J. Bucklew and G. Wise (1982): Multidimensional Asymptotic Quantization Theory with $\mathrm{r}^{\text {th }}$ Power Distortion Measures. IEEE Trans. on Information Theory, special issue on Quantization 28(2), 239-247.

[6] C. Carmona and N. Touzi (2008). Optimal Multiple Stopping and valuation of Swing Options. Mathematical Finance, 18(2), 239-268.

[7] A. Gersho and R. Gray (1982).(eds.), IEEE Trans. Info. Theory, Special issue on Quantization 28, 127-334.

[8] S. Graf and H. Luschgy (2000). Foundations of quantization for probability distributions, Lecture Notes in Mathematics, no. 1730. New York: Springer.

[9] R. Gray and D. Neuhoff (1998). Quantization, IEEE Trans. On Information Theory 44, 2325-2382.

[10] F. Guilbaud, M. Mnif and H.Pham (2013). Numerical methods for an optimal order execution problem. Journal of Computational Finance. Vol 16 Number 3, 3-45

[11] J. Jacod and A. Shiryaev (2003). Limit theorems for stochastic processes. Springer Verlag, second edition.

[12] B. Lapeyre, A. Sulem and D. Talay (2005). Simulation of financial models: Mathematical foundations and applications, to appear. 
[13] J.Neveu (1971). Martingales à temps discret, Masson, Paris, 215p.

[14] G. Pagès (1997). A space vector quantization method for numerical integration, Journal of Comput. Appl. Math., 89, 1-38.

[15] G. Pagès, H. Pham and J. Printems (2004). Optimal quantization methods and applications to numerical problems in finance. In Handbook of Computational and Numerical Methods in Finance 253-297. Birkhäuser, Boston, MA.

[16] H. Pham (1998). Optimal Stopping of Controlled Jump Diffusion Processes: A viscosity Solution Approach. Journal of Mathematical Systems, Estimation, and Control. Vol 8, No. 1,pp. 1-27

[17] www.quantize.maths-fi.com/gaussian_database 